\title{
Flux-corrected dispersion-improved CABARET schemes for linear and nonlinear wave propagation problems
}

\author{
A. Chintagunta, S.E. Naghibi, and S.A. Karabasov
}

\begin{abstract}
The new two-time-level dispersion improved CABARET scheme is developed as an upgrade of the original CABARET for improved wave propagation modelling in multiple dimensions and for nonlinear conservation laws including gas dynamics. The new upgrade retains many attractive features of the original CABARET scheme such as shock-capturing and low dissipation. It is simple for implementation in the existing CABARET codes and leads to a greater accuracy for solving linear wave propagation problems. A non-linear version of the dispersion-improved CABARET scheme is introduced to efficiently deal with contact discontinuities and shocks. The properties of the new linear and nonlinear CABARET schemes are analysed for numerical dissipation and dispersion error based on Von Neumann analysis and Pirrozolli's method. Numerical examples for one-dimensional and two-dimensional linear advection, the one-dimensional inviscid Burger's equation, and the isothermal gas dynamics problems in one and two dimensions are presented.
\end{abstract}

Keywords: wave propagation, high-resolution schemes, dissipation and dispersion analysis, shock-capturing, CABARET

\section{Introduction}

Wave propagation phenomena play an important role in fluid dynamics from geophysical flows to aerospace engineering. Modern numerical methods for wave propagation simulations face various challenges from preserving the correct phase and amplitude information for small amplitude perturbations to shock capturing for non-linear waves.

General-purpose numerical methods which were developed to deal with these problems in the literature are equally diverse. These range from the high-resolution methods based on the ideas of Flux Corrected Transport (FCT) and Total Variation Diminishing/ Bounded (TVD/B) schemes, which emphasise shock capturing while using high-order polynomial reconstructions to accurately compute linear waves (e.g. WENO, DG methods [1-4]) to the pseudo-spectral schemes which emphasise linear wave propagation through the use of elaborate dispersion and dissipation optimised numerical stencils and apply some non-linear filter dissipation for shock capturing [5].

The dispersion improved modification of the Compact Accurately Boundary-Adjusting highREsolution Technique (CABARET) [6] is an example of the second-order scheme which combines properties of both the approaches: it has a small numerical dispersion and is nondissipative (when used without the flux correction) while also permits efficient flux correction algorithms of the same type as applied in the original CABARET scheme for shock-capturing problems [7]. 
The original CABARET scheme is a compact formulation of the second-order Upwind Leapfrog (UL) [8,9] in which the conservation and flux variables are both defined as "active". Similarly to Lax-Wendroff-type methods [10], CABARET is fully discrete in space and time. Starting from the original formulation for one dimensional linear advection equation [6] CABARET was extended to aeroacoustics and turbulent flow modelling [11-13], asynchronous time stepping [14,15] and unstructured curvilinear nested grids on GPU [16] for performing computer-under-the-desk simulations of large-size problems within short turn-around solution times. At present, CABARET has found a good use in various applications from geophysical modelling [17,18] to aeroacoustics [12] and from nuclear engineering [19] to weakly compressible flows [20] and linear elasticity problems [21].

The best operating range for CABARET calculations for accuracy in terms of the least dispersion error includes Courant-Friedrichs-Lewy (CFL) numbers close to 0.3 and higher. Depending on the grid cell aspect ratio and the wave propagation direction with respect to the grid, this best range of CFL numbers can be also close to the stability limit of the scheme since the stability condition of the CABARET scheme in 3D is $\left|C F L_{x}\right|+\left|C F L_{y}\right|+\left|C F L_{z}\right|<1$. This means that for slowly travelling waves compared to the fastest waves in the simulation, the dispersion error of the CABARET scheme can be far from optimal.

In Goloviznin and Samarskii [6], a dispersion improved version of the CABARET scheme was suggested to enhance the dispersion properties of the original CABARET scheme at small CFL numbers [6]. The improvement was achieved by adding an artificial anti-dispersion term into the governing equation which corresponds to zero dissipation. The coefficient of the dispersion term is second-order with respect to the grid spacing and includes a calibration parameter. In the original publication [6], a three-time-level form of the dispersion improved CABARET scheme was suggested and its extension to non-uniform grids was provided.

Until present, applications of the dispersion improved CABARET scheme were limited to a one-dimensional linear advection equation since the original three-time-level form of the scheme is difficult for generalisations to multiple dimensions and non-linear flows. Hence, the present work is aimed at extending the dispersion improved CABARET to the compact twolevel predictor-corrector form with inclusion of the non-linear flux correction and generalisations for multi-dimensional problems and gas dynamics.

The paper is organised as the following. The standard CABARET and its dispersionimproved version are introduced in Section 2. Flux correction algorithms and a limiter for the dispersion term of the dispersion improved CABARET are described in Section 3. In Section 4, the dispersion improved CABARET is generalised to hyperbolic systems. In Section 5, the results of dispersion and dissipation analysis of the linear and nonlinear dispersion-improved CABARET schemes are presented using Von Neumann analysis [22] and Pirrozolli's method [23]. Numerical examples for linear advection, the inviscid Burger's equation, and wave propagation in gas dynamics in one and two dimensions are provided in Section 6.

\section{Linear CABARET schemes for one-dimensional scalar conservation law}

To illustrated the ideas, let's first consider the scalar one-dimensional conservation law

$$
\frac{\partial u}{\partial t}+\frac{\partial f}{\partial x}=0
$$

where $u$ and $f=f(u)$ are conservation variable and flux function respectively. Equation (1) is solved in a finite spatial domain $-L<x<L$ as an evolutionary problem in time $0<t<T$. 
The solution domain is covered by a discrete grid in space, $\Delta x_{i+1 / 2}$ and time, $\Delta t^{n}$ with introducing conservation variables and flux variables. Conservation variables are referred to the spatial cell centres $(i+1 / 2)$ which are computed at the whole and mid-time levels, $n$ and $n+1 / 2$, respectively. Flux variables, which correspond to the same transported solution variable, are referred to the spatial cell faces $i$ and are computed at the whole time levels, $n$ (Fig.1).

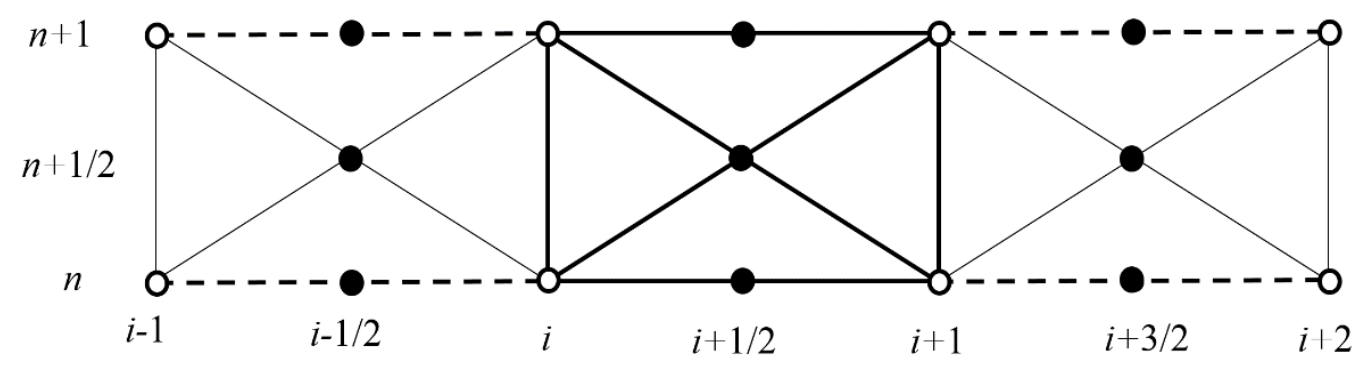

Fig. 1. Computational stencils of the CABARET (thick solid lines) and the dispersionimproved CABARET schemes (with added thick dash lines).

Starting from the known condition at the $n$th time level, the standard CABARET scheme in the predictor-corrector form can be summarised in the following three steps:

- Predictor step, conservation variables are computed using a central approximation forward in time

$$
\frac{u_{i+1 / 2}^{n+1 / 2}-u_{i+1 / 2}^{n}}{0.5 \Delta t^{n}}+\frac{f_{i+1}^{n}-f_{i}^{n}}{\Delta x_{i+1 / 2}}=0,
$$

- Extrapolator step, cell face variables at the new time level are calculated using the upwind extrapolation

$u_{i+1}^{n+1}=2 u_{i+1 / 2}^{n+1 / 2}-u_{i}^{n}$ if $\frac{d}{d u} f>0$,

$u_{i+1}^{n+1}=2 u_{i+3 / 2}^{n+1 / 2}-u_{i+2}^{n}$ if $\frac{d}{d u} f<0$,

where the sonic point $\frac{d}{d u} f=0$ generally requires a special consideration, e.g. as discussed in [24],

- Corrector step, conservative variables at the new time level are computed based on updated values of flux variables

$$
\frac{u_{i+1 / 2}^{n+1}-u_{i+1 / 2}^{n+1 / 2}}{0.5 \Delta t^{n}}+\frac{f_{i+1}^{n+1}-f_{i}^{n+1}}{\Delta x_{i+1 / 2}}=0 \text {. }
$$

The dispersion-improved version of the CABARET scheme can be introduced by modifying the definition of the flux function used in predictor step (2) and corrector step (4) so that

$$
f_{i}^{n} \rightarrow f_{i}^{n} \equiv f\left(u_{i}^{n}\right)+\delta f_{i}^{n},
$$

where the anti-dispersion correction term is given by 


$$
\begin{aligned}
& \delta f_{i}^{n}=-\frac{\mu_{i}}{\Delta \bar{x}_{i}}\left(\frac{f_{i+1}^{n}-f_{i}^{n}}{\Delta x_{i+1 / 2}}-\frac{f_{i}^{n}-f_{i-1}^{n}}{\Delta x_{i-1 / 2}}\right), \Delta \bar{x}_{i}=\frac{1}{2}\left(\Delta x_{i+1 / 2}+\Delta x_{i-1 / 2}\right), \\
& \mu_{i}=\varepsilon_{d}\left(\left(\Delta x_{i+1 / 2}\right)^{2}-3 C_{0, i+1 / 2} \Delta x_{i+1 / 2} \Delta \bar{x}_{i}+2\left(C_{0, i+1 / 2} \Delta x_{i+1 / 2}\right)^{2}\right),
\end{aligned}
$$

$\varepsilon_{d}$ is a calibration parameter which optimum value can be evaluated analytically based on the spectral analysis as will be discussed in Section 5, and $C_{0, i+1 / 2}$ is the local CFL number in cell $(\mathrm{i}+1 / 2)$ at time level $(\mathrm{n})$.

For linear advection case, e.g. $\frac{d}{d u} f=c>0$, by expressing the conservation variables via the flux variables, the predictor-corrector CABARET algorithm (2)-(5) reduces to the following three-time-level collocated finite-difference scheme

$$
\begin{aligned}
& \frac{u_{i+1}^{n+1}-u_{i+1}^{n}}{2 \Delta t^{n}}+\frac{u_{i}^{n}-u_{i}^{n-1}}{2 \Delta t^{n}}+c \frac{u_{i+1}^{n}-u_{i}^{n}}{\Delta x_{i+1 / 2}}=c\left[\frac{\mu_{i+1}}{\Delta \bar{x}_{i+1}}\left(\frac{u_{i+2}^{n}-u_{i+1}^{n}}{\Delta x_{i+3 / 2}}-\frac{u_{i+1}^{n}-u_{i}^{n}}{\Delta x_{i+1 / 2}}\right)-\frac{\mu_{i}}{\Delta \bar{x}_{i}}\left(\frac{u_{i+1}^{n}-u_{i}^{n}}{\Delta x_{i+1 / 2}}-\frac{u_{i}^{n}-u_{i-1}^{n}}{\Delta x_{i-1 / 2}}\right)\right] \\
& \mu_{i}=\varepsilon_{d}\left(\left(\Delta x_{i+1 / 2}\right)^{2}-3 c \Delta t^{n} \Delta \bar{x}_{i}+2\left(c \Delta t^{n}\right)^{2}\right),
\end{aligned}
$$

which is the same scheme as considered by Goloviznin and Samarskii [6].

For uniform grid spacings and constant time steps, the coefficient of the dispersion term becomes

$\mu=\varepsilon_{d} \Delta x^{2}\left(1-C_{0}\right)\left(1-2 C_{0}\right)$.

It is identically zero for $C_{0}=0.5$ and $C_{0}=1$ which correspond to the CFL numbers at which the original CABARET scheme is exact for linear advection equation.

Note that the computational stencil of the dispersion improved CABARET for the onedimensional advection equation involves 3 space-time cells compared to the standard CABARET which takes just 1 cell. The difference becomes less for applications which involve characteristics of opposite signs meeting at the same cell face, e.g. gas dynamics, where the dispersion improved CABARET still involves 3 cells while the standard CABARET stencil extends to 2 cells. Furthermore, when solving equations of a convection-diffusion-type, where the diffusion approximation for CABARET typically involves cell-centre variables [12], the computational stencil becomes 3 cells in each coordinate direction for both versions of the CABARET algorithm.

\section{Nonlinear CABARET schemes}

\subsection{Flux correction}

In [7], there were two flux-corrected CABARET schemes suggested. The baseline correction method corresponds to truncating the flux value computed at the extrapolation step (3) if it is found to lie outside the allowable limits in accordance with the discrete analogue of the maximum principle, e.g.

case $\frac{d}{d u} f>0$, 
$m=\min \left(u_{i}^{n}, u_{i+1 / 2}^{n}, u_{i+1}^{n}\right), M=\max \left(u_{i}^{n}, u_{i+1 / 2}^{n}, u_{i+1}^{n}\right)$,

$u_{i+1}^{n+1}=\operatorname{median}\left(m, 2 u_{i+1 / 2}^{n}-u_{i}^{n}, M\right)$,

case $\frac{d}{d u} f<0$,

$m=\min \left(u_{i+1}^{n}, u_{i+3 / 2}^{n}, u_{i+2}^{n}\right), M=\max \left(u_{i+1}^{n}, u_{i+3 / 2}^{n}, u_{i+2}^{n}\right)$,

$u_{i+1}^{n+1}=\operatorname{median}\left(m, 2 u_{i+3 / 2}^{n}-u_{i+2}^{n}, M\right)$.

The above algorithm is robust for non-linear wave problems but leads to artificial dissipation when computing linear wave propagation at high frequencies when the discrete maximum principle based on three solution points per computational cell is not very accurate. Furthermore, for small CFL numbers away from the optimum range for CABARET, this dissipation becomes even larger since in this case the flux correction is most active to smear out effects of the growing numerical dispersion error.

The second flux-correction method suggested in [7] for high-frequency wave propagation corresponds to artificially extending the maximum and minimum limits computed in (9) and (10) so that

$m \rightarrow m \equiv(1-\varepsilon \cdot \operatorname{sign}(m)) m, M \rightarrow M \equiv(1+\varepsilon \cdot \operatorname{sign}(M)) M$,

where $\varepsilon \geq 0$ is a small tuneable parameter, the limiting cases of which correspond to the baseline flux correction ( $\varepsilon=0$ ) or the linear CABARET scheme $(\varepsilon \rightarrow+\infty)$. Unfortunately, it was later found out that the optimal value of this tuneable parameter is very case dependent. Hence, instead of (11), a modified relaxed flux correction algorithm for CABARET is considered in the current publication that is based on the following modification of the maximum and minimum limits in (9) and (10):

$m \rightarrow m \equiv m-\varepsilon \cdot \delta, M \rightarrow M \equiv M+\varepsilon \cdot \delta$,

where $\delta=\frac{1}{2}(M-m)$ and $\varepsilon \geq 0$ is a tuneable parameter. This modification corresponds to a linear stretching of the solution variation as allowed by the discrete maximum principle with a constant factor so that $M-m \rightarrow(1+\varepsilon)(M-m)$. Compared to algorithm (11), the values of this parameter in (12) lying within the range of $0.2 \leq \varepsilon \leq 0.4$ lead to acceptable results for both linear advection and gas dynamics problems as will be demonstrated in Section 6 .

For strongly non-linear problems, where even a small amount of numerical oscillations is unwanted, a further refined modified relaxed correction algorithm is proposed which reduces to the baseline correction (9) and (10) in the non-linear region of large wave amplitudes (e.g. shocks) while uses the relaxation of the maximum and minimum limits in accordance with (12) when the wave amplitudes are small corresponding to a linear solution regime (e.g. acoustic wave propagation). The detection of the linear and the non-linear wave propagation regimes can be performed automatically by using the following limiter function:

$m \rightarrow m \equiv m-\varepsilon \cdot \phi(\delta) \bar{m}, M \rightarrow M \equiv M+\varepsilon \cdot \phi(\delta) \bar{m}$, 
where $\bar{m}=0.5(m+M)$ and $\phi(\delta)= \begin{cases}\delta / \bar{m} & \text { if }|\delta / \bar{m}|<<1 \\ 0 & \text { else }\end{cases}$

For practical implementation, the following threshold values can be used

$\phi(\delta)= \begin{cases}\delta / \bar{m} & \text { if }|\delta / \bar{m}|<0.001-0.01 \\ 0 & \text { else }\end{cases}$

\subsection{Dispersion term correction}

In comparison with the standard CABARET scheme, the dispersion improved CABARET (1) - (5) includes the third derivative, which is used to counteract the numerical dispersion for linear propagation. However, across a discontinuity such as a shock, the high-order derivative term becomes ill-posed, hence, defeats the original propose of using the anti-dispersion term for improving the propagation properties of the CABARET algorithm.

Therefore, for non-linear flow regimes, where

$\left|\left(f_{i+1}^{n}-f_{i}^{n}\right) / \bar{f}_{i+1 / 2}^{n}\right| \sim O(1)$, where $\bar{f}_{i+1 / 2}^{n}=0.5\left(f_{i+1}^{n}+f_{i}^{n}\right)$,

an automatic procedure is suggested to smoothly deactivate the dispersion correction. In implementations, the following detection condition of the non-linear wave region can be used

$\left|\left(f_{i+1}^{n}-f_{i}^{n}\right) / \bar{f}_{i+1 / 2}^{n}\right|>0.001-0.1$ where $\bar{f}_{i+1 / 2}^{n}=0.5\left(f_{i+1}^{n}+f_{i}^{n}\right)$.

The limiting of the dispersion term can be achieved by the following modification of the dispersion flux (6) based on the evaluation of the solution slope ratio, $r_{i}=\frac{f_{i+1}^{n}-f_{i}^{n}}{\Delta x_{i+1 / 2}}\left(\frac{f_{i}^{n}-f_{i-1}^{n}}{\Delta x_{i-1 / 2}}\right)^{-1}:$

case $\left|r_{i}\right|>1, \delta f_{i}^{n}=-\frac{\mu_{i}}{\Delta \bar{x}_{i}} \frac{f_{i}^{n}-f_{i-1}^{n}}{\Delta x_{i-1 / 2}}\left(\phi\left(r_{i}\right)-1\right)$

case $\left|r_{i}\right| \leq 1, \delta f_{i}^{n}=-\frac{\mu_{i}}{\Delta \bar{x}_{i}} \frac{f_{i+1}^{n}-f_{i}^{n}}{\Delta x_{i+1 / 2}}\left(1-\phi\left(1 / r_{i}\right)\right)$

where the limiter function $\phi(x)=\left\{\begin{array}{ll}x & \text { if }|x| \leq \alpha \\ 1 & \text { else }\end{array}\right.$.

Threshold values in the region of $1 \leq \alpha \leq 2$ ( note that $\alpha=1$ reduces the dispersion-improved scheme to the standard CABARET) lead to quite acceptable results for the problems considered in the current publication. 


\section{Extension of the dispersion-improved CABARET scheme to hyperbolic systems in multiple dimensions}

\subsection{Dispersion-improved CABARET for hyperbolic conservation laws}

Next, we consider the extension of the dispersion-improved CABARET to a system of hyperbolic equations in three dimensions

$$
\frac{\partial \mathbf{U}}{\partial t}+\frac{\partial \mathbf{F}}{\partial x}+\frac{\partial \mathbf{G}}{\partial y}+\frac{\partial \mathbf{H}}{\partial z}=0
$$

where $\mathbf{U}$ is the vector of conservation variables that consists of $m$ components, $\mathbf{F}=\mathbf{F}(\mathbf{U}), \mathbf{G}=\mathbf{G}(\mathbf{U}), \mathbf{H}=\mathbf{H}(\mathbf{U})$ are the flux vectors. $\mathbf{L}_{q}, \mathrm{q}=x, y, z$ is the left eigen-matrix which diagonalises one of the corresponding matrices $\mathbf{A}=\frac{\mathrm{d}}{\mathrm{d} \mathbf{U}} \mathbf{F}(\mathbf{U}), \mathbf{B}=\frac{\mathrm{d}}{\mathrm{dU}} \mathbf{G}(\mathbf{U}), \mathbf{C}=\frac{\mathrm{d}}{\mathrm{d} \mathbf{U}} \mathbf{H}(\mathbf{U})$ so that $\mathbf{L}_{x} \otimes \mathbf{A} \otimes \mathbf{L}_{x}^{-1}=\boldsymbol{\Lambda}_{x}=\operatorname{diag}\left\lfloor\lambda_{1 x}, \ldots, \lambda_{m x}\right\rfloor$ for example, and $\mathbf{R}_{q}=\mathbf{L}_{q} \otimes \mathbf{U}$ is the corresponding matrix of local Riemann invariants.

Following [12], the three-dimensional solution domain is covered with a hexahedral grid in space, where one conservation variable in the cell centre corresponds to several face variables defined at the face centres. Applying the divergence theorem to integrate the fluxes over $N$ faces, $\mathrm{d} \mathbf{A}_{j}, j=1, . ., N$ of the computational volume, $V_{\text {cell }}$ at the conservative predictor and corrector steps and also using the characteristic decomposition at the extrapolation step to update the flux variables, the 3D CABARET algorithm is summarised below.

- Predictor step, conservation variables are computed using a central approximation forward in time

$$
V_{\text {cell }} \frac{\mathbf{U}_{c e l l}^{n+1 / 2}-\mathbf{U}_{\text {cell }}^{n}}{0.5 \Delta t^{n}}+\sum_{j=1, N} \mathbf{F}_{j}^{n} \mathrm{~d} A_{j, x}+\sum_{j=1, N} \mathbf{G}_{j}^{n} \mathrm{~d} A_{j, y}+\sum_{j=1, N} \mathbf{H}_{j}^{n} \mathrm{~d} A_{j, z}=0,
$$

- Extrapolator step, the characteristic flux variables are computed from the conservation variables $\mathbf{R}_{q}=\mathbf{L}_{q} \otimes \mathbf{U}, q=x, y, z$ and then, for each cell face $j$, the characteristic variables at the new time level are updated using the upwind extrapolation

$R_{q, p, j}^{n+1}=2 R_{q, p, \text { upstream_cell }}^{n+1 / 2}-R_{q, p, \text { upstream_face }}^{n}, p=1, \ldots, m$,

where the location of the upstream cell centre and the upstream cell face is defined based on the sign of the corresponding eigen-speed $\lambda_{p, j}^{n+1}$; the latter is evaluated at the cell face using the information from the adjacent cell centres; after the extrapolated values of the local Riemann variables are obtained at the new time level $n+1$, their cellface values are corrected in accordance with the maximum principle; then the Riemann problem is solved to reconstruct the conservation flux variables at the new time level, $\mathbf{U}_{j}^{n+1}$ using the updated characteristic fields,

- Corrector step, conservative variables at the new time level are computed based on updated values of flux variables

$V_{\text {cell }} \frac{\mathbf{U}_{\text {cell }}^{n+1}-\mathbf{U}_{\text {cell }}^{n+1 / 2}}{0.5 \Delta t^{n}}+\sum_{j=1, N} \mathbf{F}_{j}^{n+1} \mathrm{~d} A_{j, x}+\sum_{j=1, N} \mathbf{G}_{j}^{n+1} \mathrm{~d} A_{j, y}+\sum_{j=1, N} \mathbf{H}_{j}^{n+1} \mathrm{~d} A_{j, z}=0$, 
The generalisation of the dispersion-improved version of the CABARET scheme for the onedimensional scale conservation law case (5),(6) to the 3D system can be introduced as the following modification of the vector fluxes used at predictor step (19) and corrector step (21):

$(\mathbf{F}, \mathbf{G}, \mathbf{H})_{j}^{n} \rightarrow(\mathbf{F}, \mathbf{G}, \mathbf{H})_{j}^{n} \equiv(\mathbf{F}, \mathbf{G}, \mathbf{H})\left(\mathbf{U}_{j}^{n}\right)+\delta(\mathbf{F}, \mathbf{G}, \mathbf{H})_{j}^{n}$,

where the anti-dispersion correction terms are given by

$$
\begin{aligned}
& \delta(\mathbf{F}, \mathbf{G}, \mathbf{H})_{j}^{n}=-\frac{\mu_{j}}{\Delta \bar{l}_{j}}\left(\frac{(\mathbf{F}, \mathbf{G}, \mathbf{H})_{\text {downstream_face }}^{n}-(\mathbf{F}, \mathbf{G}, \mathbf{H})_{j}^{n}}{\Delta l_{\text {downstream_cell }}^{n}}-\frac{\left.(\mathbf{F}, \mathbf{G}, \mathbf{H})_{j}^{n}-(\mathbf{F}, \mathbf{G}, \mathbf{H})_{\text {upstream_face }}^{n}\right),}{\Delta l_{\text {upstream_cell }}}\right), \\
& \Delta \bar{l}_{j}=\frac{1}{2}\left(\Delta l_{\text {downstream_cell }}+\Delta l_{\text {upstream_cell }}\right), \\
& \mu_{j}=\varepsilon_{d}\left(\left(\Delta l_{\text {downstream_cell }}\right)^{2}-3 C_{0, j, d o w n s t r e a m_{1} \text { cell }} \Delta l_{\text {downstream_cell }} \Delta \bar{l}_{j}+2\left(C_{0, j, d o w n s t r e a m_{1} \text { cell }} \Delta l_{\text {downstream_cell }}\right)^{2}\right) .
\end{aligned}
$$

In the above, $\Delta l_{\text {downstream_cell }}$ and $\Delta l_{\text {upstream_cell }}$ are the distances between the centres of the opposite cell faces, one of which is face $j$, and the downstream cell and the upstream cell, respectively. $\varepsilon_{d}$ is a calibration parameter which is evaluated from the spectral analysis. $C_{0, j, \text { downstream_cell }}$ is the local CFL number in the downstream cell at time level $n$ in the direction of the line joining the centres of the two opposite faces of the cell which includes the given face $j$ :

$C_{0, j, \text { downstream_cell }}=\frac{\max \left(\left|\lambda_{j 1}\right|, \ldots,\left|\lambda_{j m}\right|\right) \Delta t^{n}}{\Delta l_{\text {downstream_cell }}}$, where $\quad \lambda_{j, p}, p=1, . ., m$ are the corresponding characteristic speeds referred to the cell centre.

It can be seen that, for a slowly varying mean-flow field, $\mathbf{A}=\frac{\mathrm{d}}{\mathrm{d} \mathbf{U}} \mathbf{F}(\mathbf{U}) \approx$ const, $\mathbf{B}=\frac{\mathrm{d}}{\mathrm{d} \mathbf{U}} \mathbf{G}(\mathbf{U}) \approx$ const, $\mathbf{C}=\frac{\mathrm{d}}{\mathrm{d} \mathbf{U}} \mathbf{H}(\mathbf{U}) \approx$ const, fast-propagating waves (e.g. acoustic wave propagation compared to hydrodynamics for subsonic flows), and orthogonal grid $(i, j, k)$ aligned with $x, y$, and $z$ directions, the above algorithm reduces to the onedimensional dispersion improved CABARET scheme for characteristic fields $R_{q, p}, q=x, y, z$, and $p=1, . ., m$ in each coordinate direction. For example, in the $x$-direction the predictor step (19) can be re-arranged to

$$
\begin{aligned}
& \frac{\mathbf{U}_{\text {cell }}^{n+1 / 2}-\mathbf{U}_{\text {cell }}^{n}}{0.5 \Delta t^{n}}+\frac{\mathbf{F}\left(\mathbf{U}_{i+1}^{n}\right)-\mathbf{F}\left(\mathbf{U}_{i}^{n}\right)}{\Delta x_{i+1 / 2}}= \\
& =\left[\frac{\mu_{i+1}}{\Delta \bar{x}_{i+1}}\left(\frac{\mathbf{F}\left(\mathbf{U}_{i+2}^{n}\right)-\mathbf{F}\left(\mathbf{U}_{i+1}^{n}\right)}{\Delta x_{i+3 / 2}}-\frac{\mathbf{F}\left(\mathbf{U}_{i+1}^{n}\right)-\mathbf{F}\left(\mathbf{U}_{i}^{n}\right)}{\Delta x_{i+1 / 2}}\right)-\frac{\mu_{i}}{\Delta \bar{x}_{i}}\left(\frac{\mathbf{F}\left(\mathbf{U}_{i+1}^{n}\right)-\mathbf{F}\left(\mathbf{U}_{i}^{n}\right)}{\Delta x_{i+1 / 2}}-\frac{\mathbf{F}\left(\mathbf{U}_{i}^{n}\right)-\mathbf{F}\left(\mathbf{U}_{i-1}^{n}\right)}{\Delta x_{i-1 / 2}}\right)\right]+ \\
& +\mathbf{Q}_{\text {cell }}, \\
& \mathbf{Q}_{\text {cell }}=-\frac{\mathbf{G}_{j+1}^{n}-\mathbf{G}_{j}^{n}}{\Delta y_{j+1 / 2}}-\frac{\mathbf{H}_{k+1}^{n}-\mathbf{H}_{k}^{n}}{\Delta z_{k+1 / 2}}, \text { cell }=(i+1 / 2, j+1 / 2, k+1 / 2),
\end{aligned}
$$

where $\mu_{i}$ is given by (6) which involves $C_{0}=\frac{\max \left(\left|\lambda_{1, x}\right|, \ldots,\left|\lambda_{m, x}\right|\right) \Delta t^{n}}{\Delta x_{i+1 / 2}}$. 
By multiplying both sides of (24) by the left eigen matrix $\mathbf{L}_{x} \approx$ const the system of equations decouples into $m$ equations for each Riemann invariant $p=1, . ., m$ in the $\mathrm{x}$-direction:

$$
\begin{aligned}
& \frac{R_{x, p, c e l l}^{n+1 / 2}-R_{x, p, c e l l}^{n}}{0.5 \Delta t^{n}}+\lambda_{x, p} \frac{R_{x, p, i+1}^{n}-R_{x, p, i}^{n}}{\Delta x_{i+1 / 2}}= \\
& =\lambda_{x, p}\left[\frac{\mu_{i+1}}{\Delta \bar{x}_{i+1}}\left(\frac{R_{x, p, i+2}^{n}-R_{x, p, i+1}^{n}}{\Delta x_{i+3 / 2}}-\frac{R_{x, p, i+1}^{n}-R_{x, p, i}^{n}}{\Delta x_{i+1 / 2}}\right)-\frac{\mu_{i}}{\Delta \bar{x}_{i}}\left(\frac{R_{x, p, i+1}^{n}-R_{x, p, i}^{n}}{\Delta x_{i+1 / 2}}-\frac{R_{x, p, i}^{n}-R_{x, p, i-1}^{n}}{\Delta x_{i-1 / 2}}\right)\right]+ \\
& +Q_{x, p, \text { cell }}
\end{aligned}
$$

where $Q_{x, p, \text { cell }}$ is the corresponding component of matrix $\mathbf{L}_{x} \otimes \mathbf{Q}_{\text {cell }}$ and $\mu_{i}=\varepsilon_{d}\left(\left(\Delta x_{i+1 / 2}\right)^{2}-3 C_{0} \Delta x_{i+1 / 2} \Delta \bar{x}_{i}+2\left(C_{0} \Delta x_{i+1 / 2}\right)^{2}\right)$. For fast running waves $\left|\lambda_{p, x}\right| \approx \max \left(\left|\lambda_{1, x}\right|, \ldots,\left|\lambda_{m, x}\right|\right)$, the latter expression for $\mu_{i}$ is identical to (6).

\subsection{Example of the dispersion-improved CABARET scheme for 2D isothermal gas dynamics equations}

Let's consider isothermal gas equations in two dimensions written in the vector conservation law form

$\frac{\partial \mathbf{U}}{\partial t}+\frac{\partial \mathbf{F}}{\partial x}+\frac{\partial \mathbf{G}}{\partial y}=0$

where $\mathbf{U}=\left(\begin{array}{c}\rho \\ \rho u \\ \rho v\end{array}\right), \mathbf{F}=\left(\begin{array}{c}\rho u \\ \rho u^{2}+\rho c^{2} \\ \rho u v\end{array}\right)$ and $\mathbf{G}=\left(\begin{array}{c}\rho u \\ \rho u v \\ \rho v^{2}+\rho c^{2}\end{array}\right)$,

and where $\rho, u, v, p$ and $c=$ const $>0$ are density, $x$-velocity, $y$-velocity, pressure and sound speed, respectively.

The quasi-linear form of the same equations is

$$
\frac{\partial \mathbf{V}}{\partial t}+\mathbf{A} \frac{\partial \mathbf{V}}{\partial x}+\mathbf{B} \frac{\partial \mathbf{V}}{\partial y}=0
$$

where $\mathbf{A}=\left(\begin{array}{ccc}u & \rho & 0 \\ c^{2} / \rho & u & 0 \\ 0 & 0 & u\end{array}\right), \mathbf{B}=\left(\begin{array}{ccc}v & 0 & \rho \\ 0 & v & 0 \\ c^{2} / \rho & 0 & v\end{array}\right)$, and $\mathbf{V}=\left(\begin{array}{c}\rho \\ u \\ v\end{array}\right)$

The corresponding characteristic speeds and the Riemann invariants in this case are:

$\lambda_{1}^{A}=u-c, \lambda_{2}^{A}=u, \lambda_{3}^{A}=u+c, R_{1}^{A}=u-c \ln \rho, R_{2}^{A}=v, R_{3}^{A}=u+c \ln \rho$ for the x-direction

and 
$\lambda_{1}^{B}=v-c, \lambda_{2}^{B}=v, \lambda_{3}^{B}=v+c ; R_{1}^{B}=v-c \ln \rho, R_{2}^{B}=u, R_{3}^{B}=v+c \ln \rho$ for the y-direction.

The predictor-corrector steps of the algorithm amount to the 2D versions of (19), (21), (22), and (23), where the conservation variables and fluxes are defined in accordance with (27).

Details of the characteristic decomposition at the extrapolation step (20) are summarised below for the case of orthogonal grid $(i, j)$ that is aligned with coordinates $(x, y)$. The indexing convention is such that point $(i+1 / 2, j+1 / 2)$ corresponds to the cell centre and points $(i+1, j+1 / 2)$, $(i, j+1 / 2)$ and $(i+1 / 2, j+1),(i+1 / 2, j)$ correspond to the cell face centres in the $x$ - and $y$-directions, respectively.

\section{Fluxes in the $x$-direction}

1. Start from the solution variables $\rho, u, v, p$ computed at the cell centres (time level $n$ and $n+1 / 2)$ and faces (time level $n$ ).

2. Calculate the Riemann variables in the same cell centre and face points using (29).

3. Update the cell-face values of the Riemann invariants

$R_{p, i+1, j+1 / 2}^{n+1}=2 R_{p, i+1 / 2, j+1 / 2}^{n+1 / 2}-R_{p, i, j+1 / 2}^{n}$ if $\lambda_{p, i+1, j+1 / 2}^{n+1 / 2} \geq 0$,

$R_{p, i+1, j+1 / 2}^{n+1}=2 R_{p, i+3 / 2, j+1 / 2}^{n+1 / 2}-R_{p, i+2, j+1 / 2}^{n}$ else,

where $\lambda_{p, i+1, j+1 / 2}^{n+1 / 2}=0.5\left(\lambda_{p, i+1 / 2, j+1 / 2}^{n+1 / 2}+\lambda_{p, i+3 / 2, j+1 / 2}^{n+1 / 2}\right)$ and $p=1,2,3$.

For subsonic flow cases considered in the numerical examples section, $p=3$ corresponds to a right running pressure wave with $\lambda_{3, i+1, j+1 / 2}^{n+1 / 2} \geq 0$ and $p=1$ corresponds to a left running pressure wave.

4. Calculate the maximum and minimum values of each Riemann invariant for step $n+1$ in accordance with the one-dimensional maximum principle

$$
\begin{aligned}
& (m, M)_{p, i+1, j+1 / 2}^{n}=(\min , \max )\left(R_{p, i, j+1 / 2}^{n}, R_{p, i+1 / 2, j+1 / 2}^{n}, R_{p, i+1, j+1 / 2}^{n}\right) \text { if } \lambda_{p, i+1, j+1 / 2}^{n+1 / 2} \geq 0, \\
& (m, M)_{p, i+1, j+1 / 2}^{n}=(\min , \max )\left(R_{p, i+1, j+1 / 2}^{n}, R_{p, i+3 / 2, j+1 / 2}^{n}, R_{p, i+2, j+1 / 2}^{n}\right) \text { else, }
\end{aligned}
$$

with taking into account the source terms due to the derivatives in the other direction (e.g. based on (25))

$(m, M)_{p, i+1, j+1 / 2}^{n} \equiv(m, M)_{p, i+1, j+1 / 2}^{n}+Q_{p, i+1, j+1 / 2}^{n} \cdot \Delta t^{n}$,

where $Q_{p, i+1, j+1 / 2}^{n}=\frac{R_{p, i+1 / 2, j+1 / 2}^{n+1 / 2}-R_{p, i+1 / 2, j+1 / 2}^{n}}{0.5 \Delta t^{n}}+\lambda_{p, i+1 / 2 . j+1 / 2} \frac{R_{p, i+1, j+1 / 2}^{n}-R_{p, i, j+1 / 2}^{n}}{\Delta x_{i+1 / 2}}$

5. For the flux correction with relaxation, use (11), (12), (13), (14) to further modify the values $(m, M)_{p, i+1, j+1 / 2}^{n}$ computed.

6. Truncate the new cell face values of the Riemann variables if they are found outside of the allowable range of the maximum principle following (9) and (10). 
7. Reconstruct new flux variables at the new time level from the characteristic fields at each cell face point:

$u=0.5\left(R_{1}^{A}+R_{3}^{A}\right), v=R_{2}^{A}, \rho=\exp \left(0.5\left(R_{3}^{A}-R_{1}^{A}\right) / c\right)$.

The update of the cell-face flux variables in the $y$-direction follows the same procedure.

\section{Dissipation and dispersion analysis of linear and nonlinear dispersion-improved CABARET schemes}

\subsection{Linear spectral analysis}

Following [6], Von Neumann linear analysis [22] is applied to the dispersion improved CABARET scheme for linear advection equation in the three-time-level form (7), which in the case of constant grid spacing and time step reduces to

$\frac{u_{j}^{n+1}-u_{j}^{n}}{2 \Delta t}+\frac{u_{j-1}^{n}-u_{j-1}^{n-1}}{2 \Delta t}+c \frac{u_{j}^{n}-u_{j-1}^{n}}{\Delta x}=\frac{c \mu}{\Delta x^{3}}\left[\left(u_{j+1}^{n}-2 u_{j}^{n}+u_{j-1}^{n}\right)-\left(u_{j}^{n}-2 u_{j-1}^{n}+u_{j-2}^{n}\right)\right]$

Due to linearity of the governing equation, the partial solution is sought for in the travelling wave form $u_{j}^{n}=e^{i(\omega n \Delta t+k j \Delta x)}$ for each wavenumber $k$ and frequency $\omega$

$$
\begin{aligned}
& e^{-i k j \Delta x} \frac{e^{i \omega(n+1) \Delta t}-e^{i \omega n \Delta t}}{2 \Delta t}+e^{-i k(j-1) \Delta x} \frac{e^{i \omega n \Delta t}-e^{i \omega(n-1) \Delta t}}{2 \Delta t}+c e^{i \omega n \Delta t} \frac{e^{-i k j \Delta x}-e^{-i k(j-1) \Delta x}}{\Delta x} \\
& =\frac{\mu c}{\Delta x^{3}} e^{i \omega n \Delta t}\left[\left(e^{-i k(j+1) \Delta x}-2 e^{-i k j \Delta x}+e^{-i k(j-1) \Delta x}\right)-\left(e^{-i k j \Delta x}-2 e^{-i k(j-1) \Delta x}+e^{-i k(j-2) \Delta x}\right)\right],
\end{aligned}
$$

which yields the following characteristic equation

$e^{2 i \omega \Delta t}-\left[\left(1-2 C_{0}\right)\left(1-e^{i k \Delta x}\right)+2 \frac{\mu}{\Delta x^{2}} C_{0}\left(e^{-i k \Delta x}-3+3 e^{i k \Delta x}-e^{2 i k \Delta x}\right)\right] e^{i \omega \Delta t}-e^{i k \Delta x}=0$,

The roots of the characteristic equation are

$e^{i \omega \Delta t}=e^{i k \Delta x / 2} \frac{a i+\sqrt{-a^{2}+4}}{2}=e^{i k \Delta x / 2} e^{i \theta}$,

where $a=\left[-2\left(1-2 C_{0}\right) \sin \frac{k \Delta x}{2}+16 \frac{\mu}{\Delta x^{2}} C_{0} \sin ^{3} \frac{k \Delta x}{2}\right], \theta=\arcsin \frac{a}{2}$, and $\theta$ will be real if $4-a^{2} \geq 0$

. Substituting $\mu$ from equation (7) and replacing $x$-dependent terms by their maxima, the necessary condition for the dispersion-improved CABARET scheme to be non-dissipative will become

$-1 \leq-\left(1-2 C_{0}\right)+8 \varepsilon_{d}\left(1-C_{0}\right)\left(1-2 C_{0}\right) C_{0} \leq 1$.

In order to find $\varepsilon_{d}$ values satisfying the above condition, the behaviour of the cubic polynomial $f\left(C_{0}\right)=\left(1-2 C_{0}\right)\left[-1+8 \varepsilon_{d}\left(1-C_{0}\right) C_{0}\right]$ when $0 \leq C_{0} \leq 1$ is studied next. It passes 
through three fixed points regardless of $\varepsilon_{d}\left(f(1)=-f(0)=1, f\left(\frac{1}{2}\right)=0\right)$ with its inflexion point always at $C_{0}=\frac{1}{2}$. Furthermore, we can differentiate it as

$f^{\prime}\left(C_{0}\right)=48 \varepsilon_{d} C_{0}^{2}-48 \varepsilon_{d} C_{0}+2+8 \varepsilon_{d}$

and find the roots of $f^{\prime}\left(C_{0}\right)$ according to

$C_{0}^{1,2}=\frac{1}{2}\left(1 \pm \sqrt{\frac{2 \varepsilon_{d}-1}{6 \varepsilon_{d}}}\right)$

Due to the symmetry of the polynomial with respect to its inflexion point, there will be only three possible cases in which $f\left(C_{0}\right)$ remains between -1 and 1 as illustrated in Fig. 2.

- Case I: $f\left(C_{0}\right)$ is monotonic in the whole domain $\left(C_{0}^{1,2} \notin \square\right)$.

- Case II: $f\left(C_{0}\right)$ is monotonic when $0 \leq C_{0} \leq 1\left(C_{0}{ }^{1} \leq 0 \wedge C_{0}{ }^{2} \geq 1\right)$.

- Case III: $f\left(C_{0}\right)$ is not monotonic when $0 \leq C_{0} \leq 1$ but its extremum values are between -1 and $1\left(-1 \leq f\left(C_{0}^{1,2}\right) \leq 1\right)$.

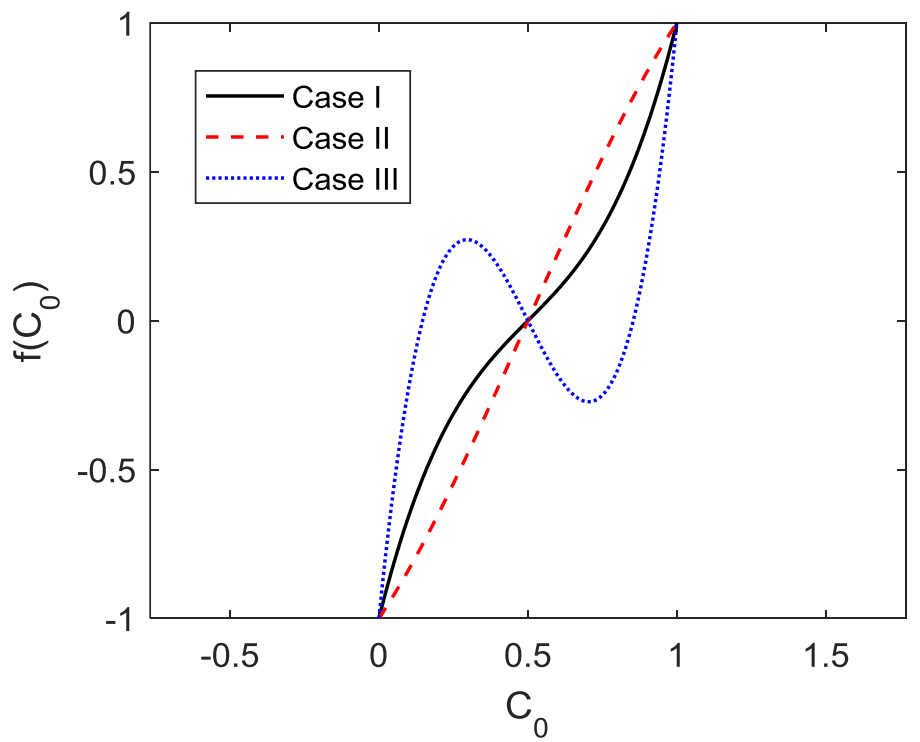

Fig. 2. Three possible cases for $f\left(C_{0}\right)$ to guarantee the stability of the dispersionimproved CABRET scheme.

Imposing the above conditions, the resulting allowed range for $\varepsilon_{d}$ will be

$D_{I} \cup D_{I I} \cup D_{I I I}=[0,0.5) \cup[-0.25,0) \cup[0.5,2]=[-0.25,2]$.

It should be noted that if the above condition is satisfied, dispersion-improved CABARET scheme will be non-dissipative $\left(\left|e^{i \omega \Delta t}\right|=1\right)$. Hence, the only source of error in $e^{i \omega \Delta t}$ will be the difference of its phase from $c k \Delta t$ 


$$
e_{\text {phase }}=\arg \left(e^{i c k \Delta t}\right)-\arg \left(e^{i(k \Delta x / 2+\theta)}\right)=k \Delta x\left(C_{0}-1 / 2\right)-\theta
$$

which can be normalized by the phase, $c k \Delta t$ to obtain expression for the relative dispersion error

$$
e_{\text {dispersion }}=1-\frac{1}{2 C_{0}}-\frac{\theta}{C_{0} k \Delta x}
$$

By further substituting the definition of $\theta$ in the phase error, the final expression for the dispersion error is obtained:

$$
e_{\text {phase }}=k \Delta x\left(C_{0}-1 / 2\right)-\arcsin \left[-\left(1-2 C_{0}\right) \sin \frac{k \Delta x}{2}+8 \varepsilon_{d}\left(1-C_{0}\right)\left(1-2 C_{0}\right) C_{0} \sin ^{3} \frac{k \Delta x}{2}\right] .
$$

To find the optimum value of $\varepsilon_{d}$, the overall absolute value of phase error should be minimized over a range of $C_{0}$ and $k \Delta x$ i.e.

$$
\frac{\mathrm{d}}{\mathrm{d} \varepsilon_{d}} \int_{0}^{1} \int_{0}^{\frac{2 \pi}{p p w}}\left|e_{\text {phase }}\right| \mathrm{d}(k \Delta x) \mathrm{d} C_{0}=0
$$

where $p p w$ stands for number of points per wavelength of the numerical wave resolution. To find the optimum value of the anti-dispersion coefficient, one should minimize the above integral using its geometrical properties as a volume integral enclosed by the surface $e_{\text {phase }}$ and $C_{0}-k \Delta x$ plane. Since the surface $e_{\text {phase }}$ passes through three parallel lines $C_{0}=0,1 / 2$ and 1 and it is symmetric with respect to the one in the middle, we can minimize the enclosed volume by making its tangent plane horizontal along the line $C_{0}=1 / 2$.

$$
\left.\frac{\mathrm{d}\left|e_{\text {phase }}\right|}{\mathrm{d} C_{0}}\right|_{C_{0}=1 / 2}=0 \text {, }
$$

which gives

$$
k \Delta x-2 \sin \frac{k \Delta x}{2}+4 \varepsilon_{d} \sin ^{3} \frac{k \Delta x}{2}=0,
$$

and for $\varepsilon_{d}$ we have

$$
\varepsilon_{d}=\frac{2 \sin \frac{k \Delta x}{2}-k \Delta x}{4 \sin ^{3} \frac{k \Delta x}{2}} .
$$

The above equation gives the optimum value of $\varepsilon_{d}$ parameter to minimize the integral in eq. (48) as a function the numerical grid resolution. Phase error distribution versus $C_{0}$ and $k \Delta x$ are shown in Figs. 3-6 and compared for three constant values of $\varepsilon_{d}$ and its optimized value from (49). Fig. 6 shows the optimum value of $\varepsilon_{d}$ approaching -1/12 as $p p w$ goes to infinity. This value is close to -0.08 originally suggested by Goloviznin and Samarskii [6] who studied the behaviour of the dispersion surfaces of numerical scheme (35) numerically for a range of 
parameter $\varepsilon_{d}$. It is the parameter value -0.08 that will be used in all further calculations with the dispersion improved CABARET scheme in the rest of the paper.

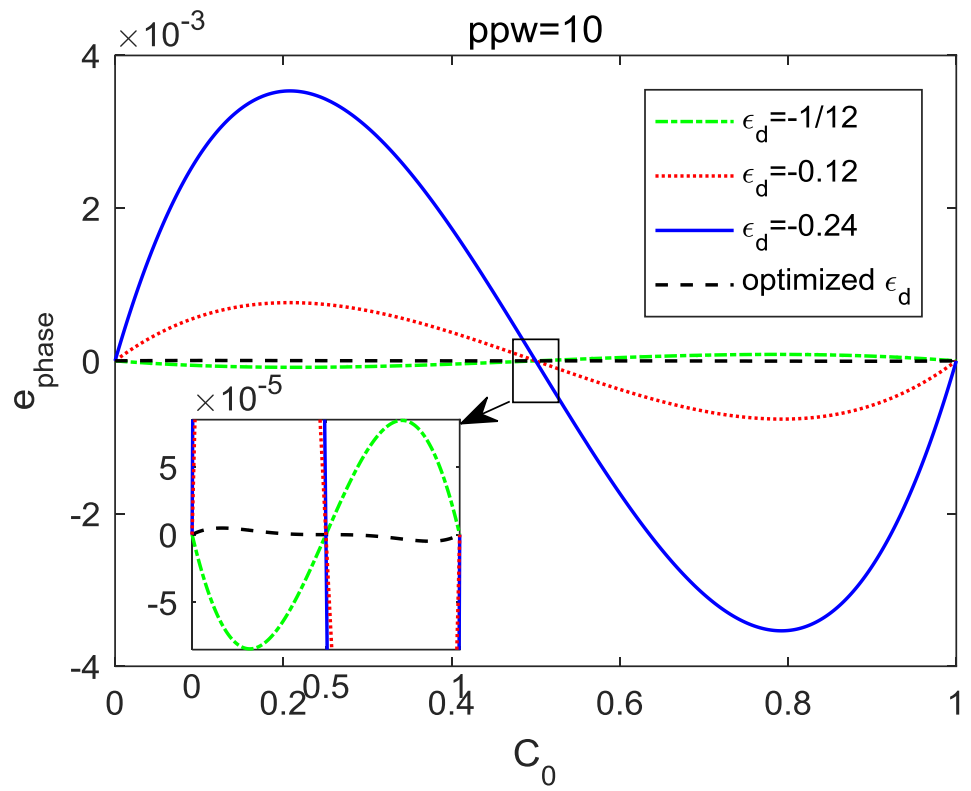

Fig. 3. Phase error distribution vs. $C_{0}$ for different values of values of $\varepsilon_{d}$ in $k \Delta x=2 \pi / 10$.

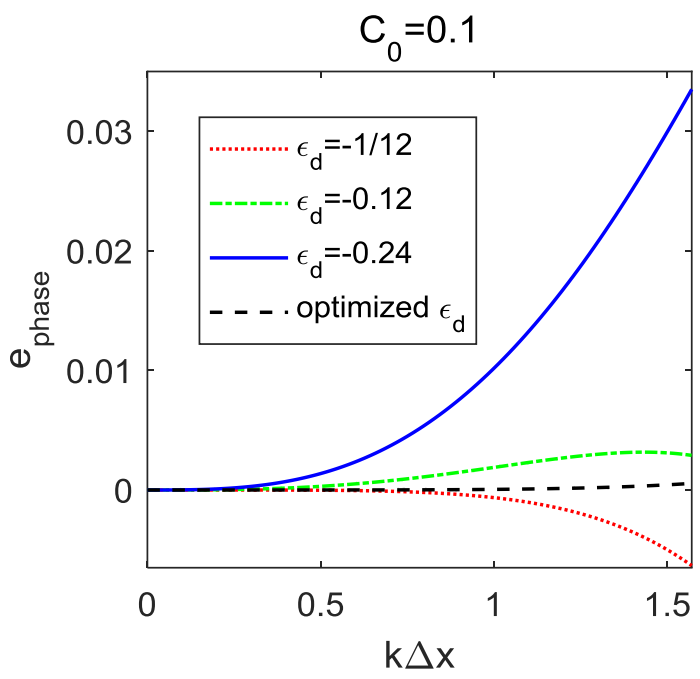

Fig. 4. Phase error distribution vs. $k \Delta x$ for different values of values of $\varepsilon_{d}$ in $C_{0}=0.1$. 


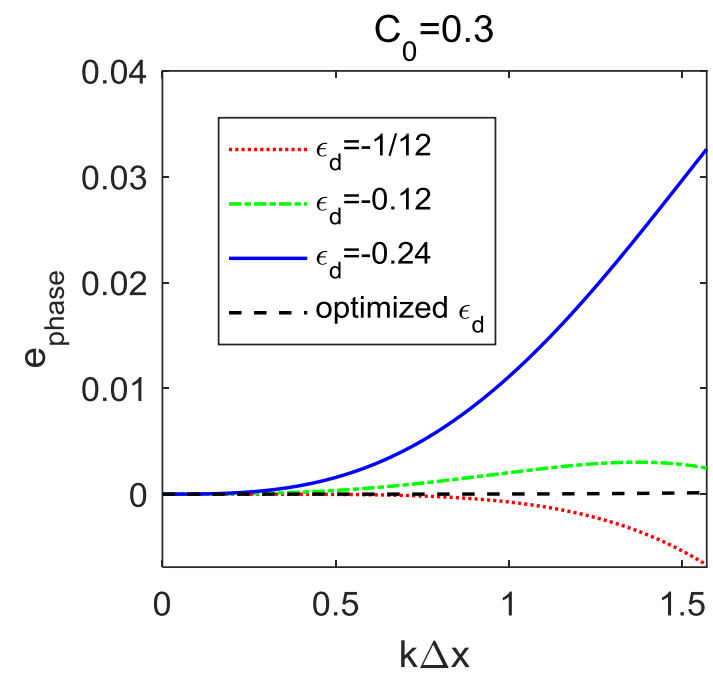

Fig. 5. Phase error distribution vs. $k \Delta x$ for different values of values of $\varepsilon_{d}$ in $C_{0}=0.3$.

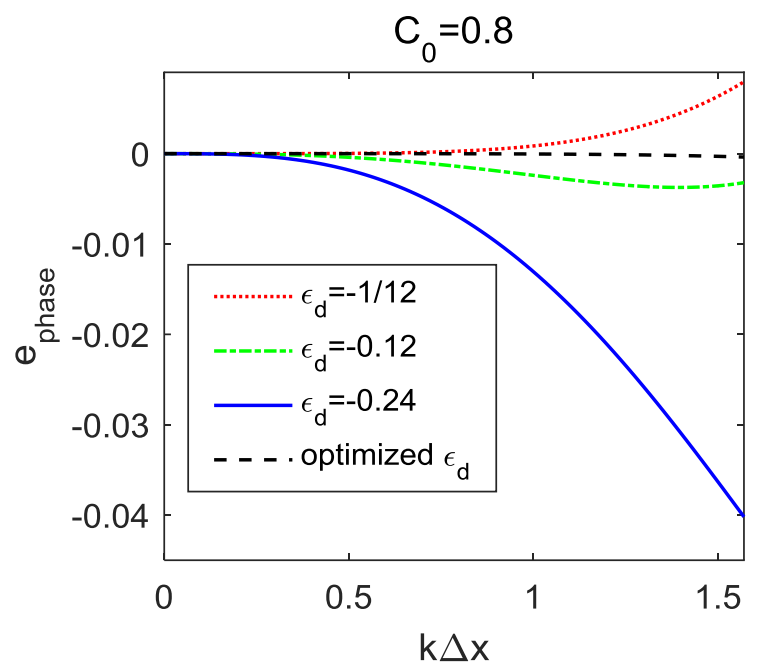

Fig. 6. Phase error distribution vs. $k \Delta x$ for different values of values of $\varepsilon_{d}$ in $C_{0}=0.8$.
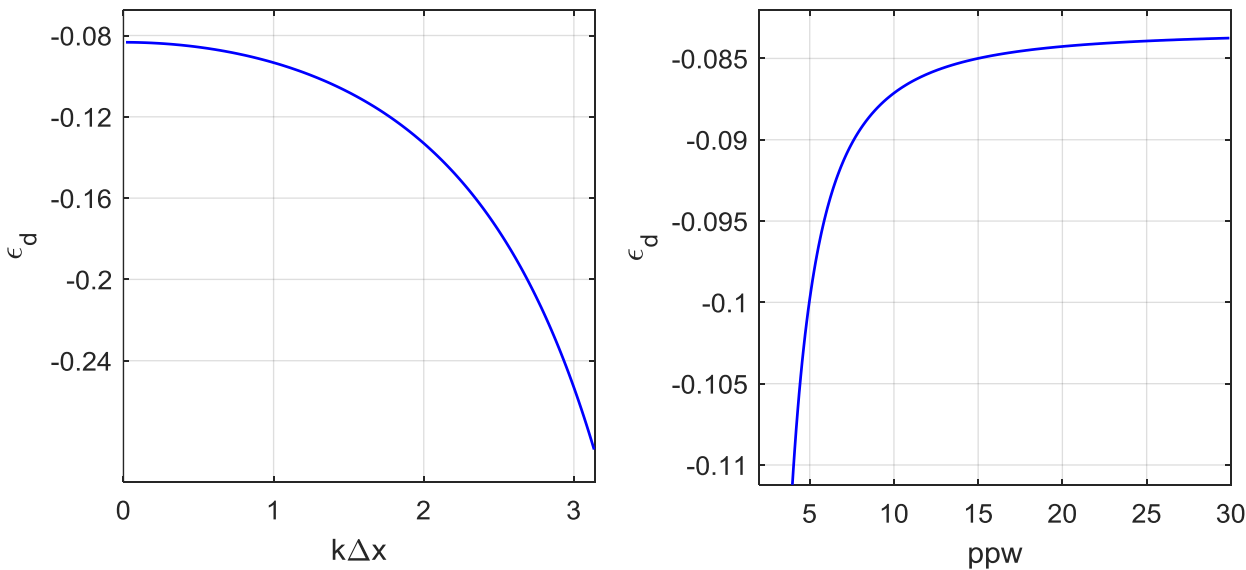

Fig. 7. Optimum distribution of dispersion parameter $\varepsilon_{d}$ vs. $k \Delta x$ and ppw. 


\subsection{Numerical spectral analysis}

For the non-linear CABARET schemes considered in section 4, the classical Von Neumann analysis doesn't apply. Alternatively, numerical experiments are suggested in the literature for determining the stability regions of nonlinear schemes such as ADER and ADER-WAF [25]. Here, we use the numerical dissipation and dispersion analysis from Pirrozolli [23]. In [26], a similar analysis was used to analyse the dissipation and dispersion properties of the standard CABARET scheme with the full flux correction (10), which results we find consistent with the results of our analysis presented below.

The idea of the non-linear dissipation and dispersion analysis method [23] is to calculate the amplitude and the phase of the solution of the one-dimensional linear advection equation with periodic boundary conditions numerically for a range of numerical wave resolutions, $k \Delta x$. The corresponding amplitude and the phase errors can be computed using the analytical solution for wave frequency at the given wavenumber and the numerical wave solution at control time $t$. The analytical solution for frequency can be expressed as a function of the amplitude and phase of the corresponding Fourier harmonic $\hat{u}_{k}(t)=\left|\hat{u}_{k}(t)\right| \exp \left(i \phi_{k}(t)\right)$ at time $t$, where $t=0$ corresponds to the initial time:

$\omega=-\frac{i}{t} \ln \left(\frac{\left|\hat{u}_{k}(t)\right|}{\left|\hat{u}_{k}(0)\right|}\right)+\frac{\phi_{k}(t)-\phi_{k}(0)}{t}$.

The exact solution of the linear advection problem is self-similar, hence, the choice of control time, $t$ is not very important. However, when computing (50) numerically, e.g. from the amplitude and the phase of the numerical solution at a wave crest, the control time $t \rightarrow t^{n}=n \Delta t$ becomes a calibration parameter of the method and needs to be taken into account so that the numerical frequency obtained from (50) doesn't depend on this parameter strongly. For example, too small values or too large values of parameter $n$ should be avoided because of the uncertainty of the wave position within the discrete grid spacing and the error escalation that corrupts the initial profile shape, respectively.

In the current work, the dissipation and the dispersion properties of the standard CABARET and the dispersion improved CABARET schemes with the full correction (10) and the modified relaxed correction (12) at $\varepsilon=0.2$ are evaluated. The numerical solution is computed for one period of the initial wave profile, which corresponds to a fixed wavenumber $k$ so that the wavelength $2 \pi / k$ is equal to the computational domain size. A series of computations is performed by covering the computational domain with uniform grids of variable resolution $\Delta x$ so that the reduced wavenumber $k \Delta x$ is varied in the desired range. The CFL number is equal to 0.1 in all cases and tests for several calibration parameters $n=t^{n} / \Delta t$ are performed to establish a range of this parameter which leads to a weak dependence of the final result on the parameter value (Fig. 8).

Results of the nonlinear dissipation and dispersion analysis at $n=65$ for CABARET schemes with full flux correction and modified relaxed flux correction are compared in Figs. 9 and 10, respectively. In each figure, three schemes are compared based the existence of the second-order anti-dispersion term: standard schemes, dispersion-improved schemes, and 
dispersion improved schemes with a nonlinear limiter. Compared to the full flux correction (fig.9a), the use of the modified flux correction leads to a factor of 2 reduction of the dissipation error at high wavenumbers (fig.10a). Inclusion of the anti-dispersion term reduces the dispersion error of the CABARET scheme at high wavenumbers compared to the baseline CABARET and the introduction of an anti-dispersion term limiter still leads to some accuracy improvement compared to the baseline method (figs.9b and 10b). The improvement in accuracy of the dispersion improved scheme over the standard CABARET method is particularly noticeable for numerical resolutions of about $8 \mathrm{ppw}$ (reduced wave number $k \Delta x \sim 0.8$ ) in case the modified relaxed flux correction is applied that allows propagating short waves with a less dissipation compared to the full flux correction (fig.10b).

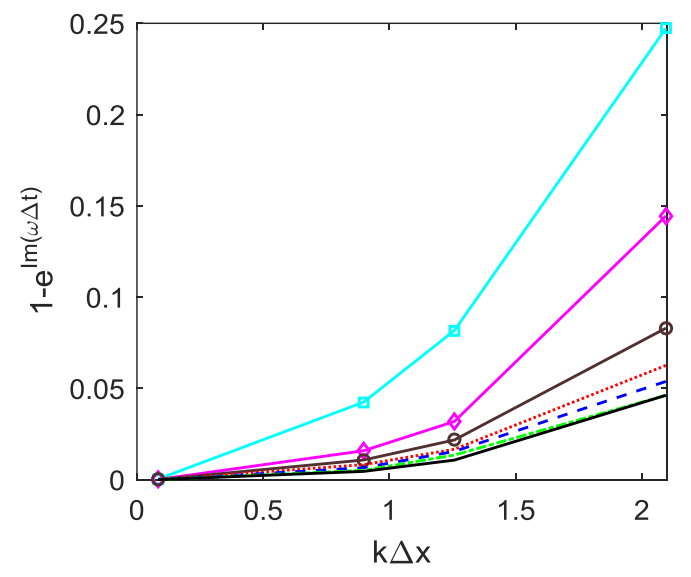

(a)

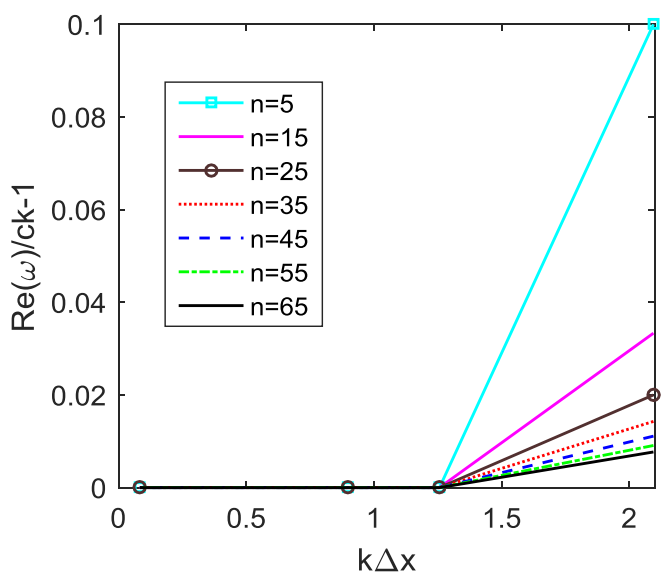

(b)

Fig. 8. Weak dependence of the dissipation (a) and dispersion (b) error of the dispersion improved CABARET scheme with the modified relaxed flux correction at $\varepsilon=0.2$ on the numerical control time parameter.

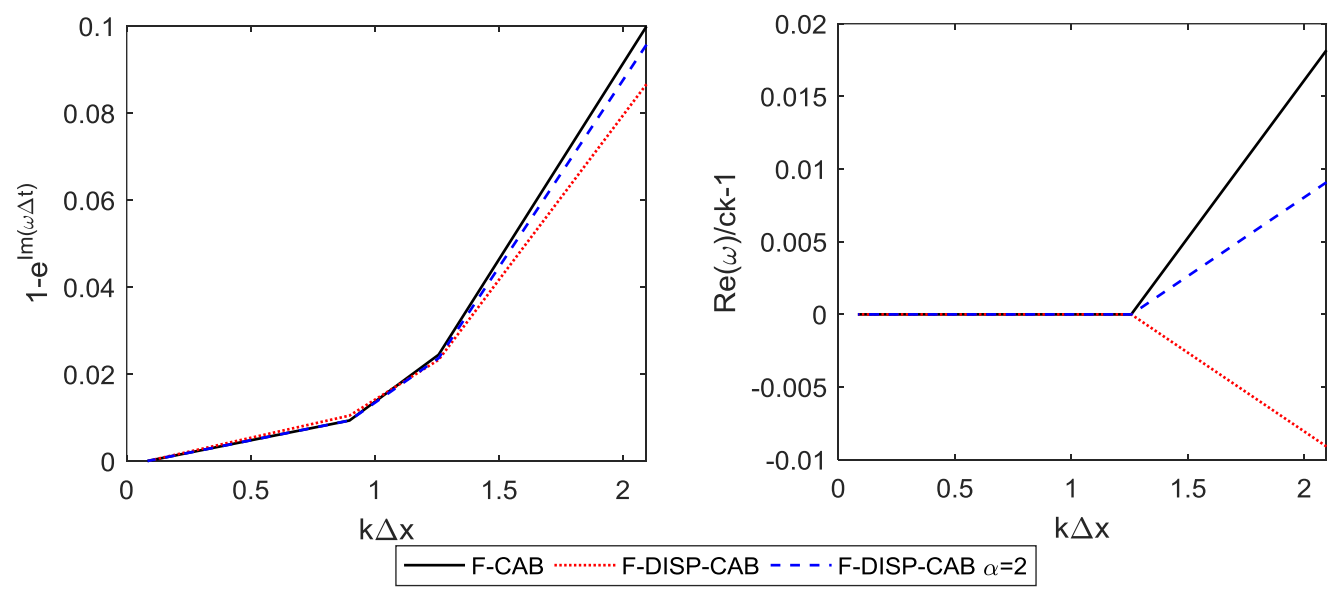

(a)

(b)

Fig. 9. Dissipation and dispersion properties of the CABARET schemes with full flux correction: comparison of the dissipation (a) and dispersion (b) errors as functions of the dimensionless wavenumber for the standard CABARET scheme, the dispersion improved 
CABARET scheme, and the dispersion improved CABARET scheme with the limiter $(\alpha=2$ ) .

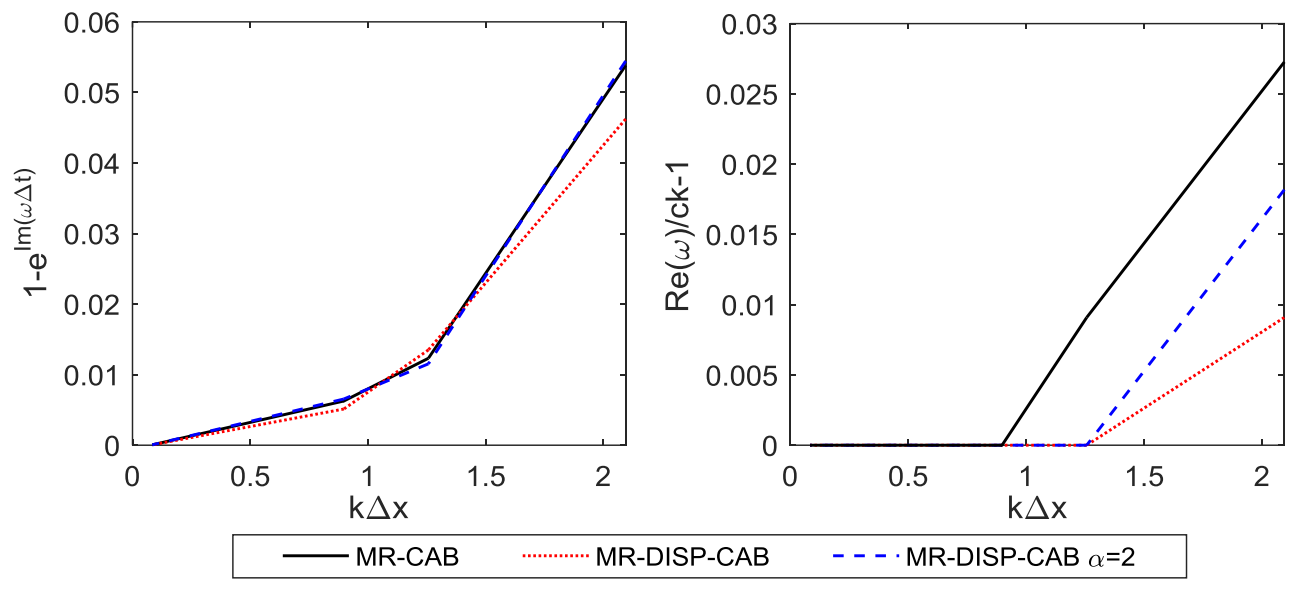

(a)

(b)

Fig. 10. Dissipation and dispersion properties of the CABARET schemes with the modified relaxed full flux correction at $\varepsilon=0.2$ : comparison of the dissipation (a) and dispersion (b) errors as functions of the dimensionless wavenumber for the standard CABARET scheme, the dispersion improved CABARET scheme, and the dispersion improved CABARET scheme with the limiter $(\alpha=2)$.

\section{Numerical Examples}

\subsection{D Linear advection}

For the first test case, one-dimensional linear advection equation is considered which corresponds to setting $f=c u$ in (1), where $c=1$ in a large domain with periodic boundary conditions.

First, smooth initial data in the form of a sine wave modulated by a Gaussian profile from [27] are considered:

$$
\begin{aligned}
& u(x, 0)=\sin (48 \pi(x / L-1 / 4)) \exp \left(-\log 2(32(x / L-1 / 4) / 3)^{2}\right), 0 \leq x \leq L / 2, \\
& u(x, 0)=0, L / 2<x \leq L .
\end{aligned}
$$

Figure 11 shows comparisons of the standard CABARET and the dispersion improved CABARET solutions without any correction applied with the analytical solution at control time $t=100$. A uniform computational grid is used that contains 200 cells, which corresponds to about 8 points per wavelength ( $p p w$ ) for resolving the sine wave. For this case, the dispersion improved CABARET solution practically coincides with the exact solution while the standard CABARET shows a notable phase error.

Table 1 shows comparisons of the numerical error convergence in L1 norm for the same test case for the following versions of the dispersion improved CABARET scheme: no correction 
(P-DISP-CAB), the scheme with the baseline flux correction (10) (F-DISP-CAB), and the scheme with the modified relaxed flux correction at $\varepsilon=0.2$ (12) (MR-DISP-CAB).

For the fine grids, the schemes with no correction and the modified relaxed correction show the rate of convergence close to 2, which corresponds to the theoretical order of approximation of the linear CABARET schemes. The scheme with the full baseline flux correction shows the rate of convergence close to 1.6-1.7 that is similar to the convergence rates of the flux-corrected CABARET scheme reported in [7].

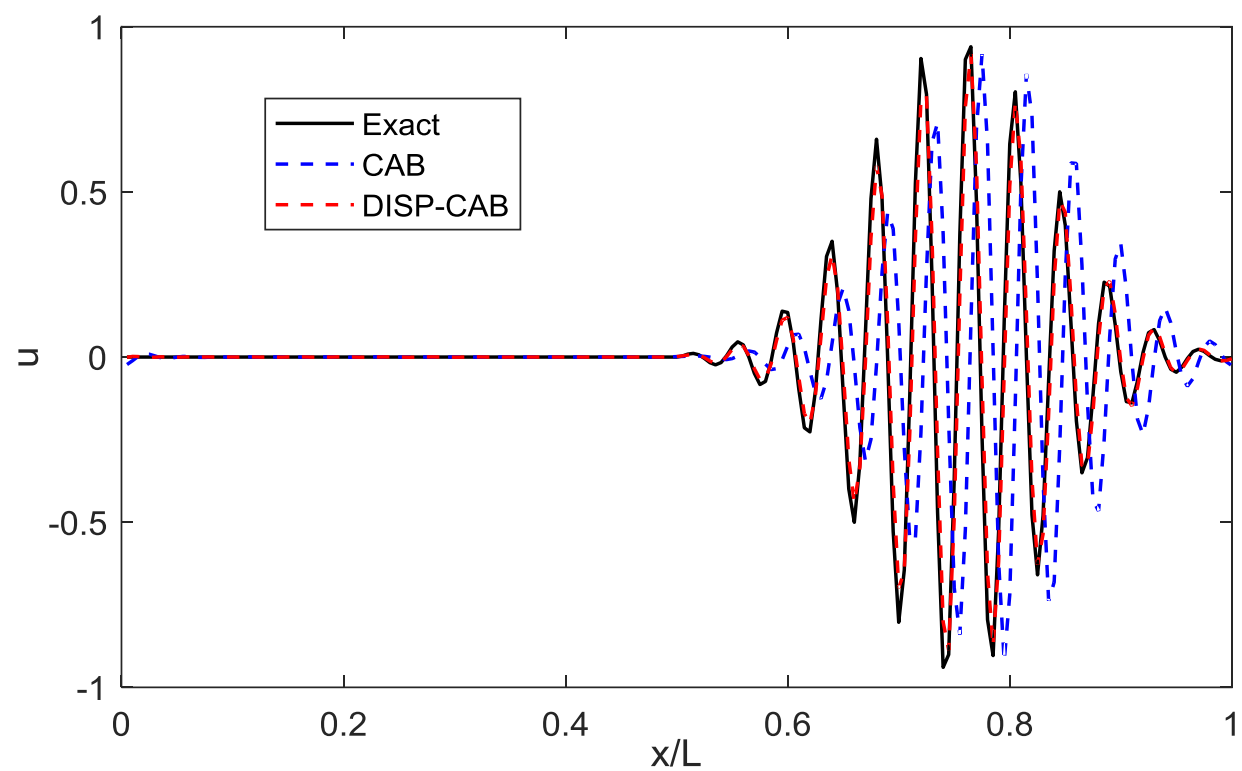

Fig. 11. Linear advection of a high-frequency wave modulated by a Gaussian: the dispersion improved CABARET (DISP-CAB) against the standard CABARET solution (CAB).

\begin{tabular}{|l|l|l|l|l|l|l|}
\hline grid size & P-DISP-CAB & order & F- DISP-CAB & order & MR- DISP -CAB & order \\
\hline 6400 & $1.323 \mathrm{e}-05$ & 1.982 & $6.54 \mathrm{e}-04$ & 1.705 & $7.51 \mathrm{e}-05$ & 2.17 \\
\hline 3200 & $5.19 \mathrm{e}-05$ & 1.995 & 0.0019 & 1.654 & $3.52 \mathrm{e}-04$ & 2.13 \\
\hline 1600 & $2.07 \mathrm{e}-04$ & 2.03 & 0.0052 & 1.681 & 0.0016 & 1.94 \\
\hline 800 & $8.48 \mathrm{e}-04$ & 2.14 & 0.0147 & 1.650 & 0.0060 & 1.83 \\
\hline 400 & 0.0039 & 2.47 & 0.0400 & 1.558 & 0.0201 & 1.439 \\
\hline 200 & 0.0237 & & 0.0971 & & 0.0416 & \\
\hline
\end{tabular}

Table 1. Grid convergence for the dispersion- improved CABARET schemes without correction (P-DISP-CAB), with the modified relaxed correction (MR- DISP-CAB), and with full correction (F- DISP-CAB) in the linear advection test of a high-frequency wave modulated by a Gaussian. 
The second initial distribution corresponding to the same linear advection equation is a rectangular pulse appended by a small amplitude high-frequency wave given by

$$
\begin{aligned}
& u(x, 0)=1+0.05 \cos (80 \pi x / L),-L \pi / 20 \leq x \leq L \pi / 20, \\
& u(x, 0)=0.05 \cos (80 \pi x / L),-L / 2 \leq x<-L \pi / 20 \text { or } L \pi / 20<x \leq L / 2 .
\end{aligned}
$$

The control time is 100 like in the first test case and boundary conditions considered to be periodic. For this initial condition, the following three scheme were compared: (i) the standard CABARET with the full correction (10), (ii) the dispersion-improved CABARET with the modified relaxed correction (12) at $\varepsilon=0.2$, and (iii) the popular fourth-order Dispersion Relation Preserving (DRP) scheme [28] combined with the fourth-order Runge-Kutta method in time. The CFL number is the same and equal to 0.2 for all three schemes.

Figure 12 shows comparisons of three numerical solutions with the analytical solution for a uniform grid of 400 cells which corresponds to the high-frequency wave resolution of 10 p.p.w. It can be seen that the standard CABARET scheme with the full flux correction captures the rectangular profile well but leads to some smearing of the high-frequency wave. DRP scheme seems to capture the phase of high-frequency wave well but leads to notable numerical oscillations which are not just confined to the region of the discontinuity but are spread around the entire solution domain. The dispersion-improved CABARET solution with the modified relaxed correction shows the best agreement with the analytical solution by capturing the rectangular profile without any noticeable wiggles and resolving the high-frequency wave at the same time.

Numerical grid convergence results for this case are shown in Table 2 for the dispersionimproved CABARET with the modified relaxed correction (MR-CAB-DISP) and for the DRP scheme. For both schemes, the L1 error norm is used. Because of the discontinuous initial profile the rate of convergence is less than 1 for both methods. Still, the error of the dispersionimproved CABARET scheme is consistently lower and its rate of convergence is consistently higher compared to the DRP results.
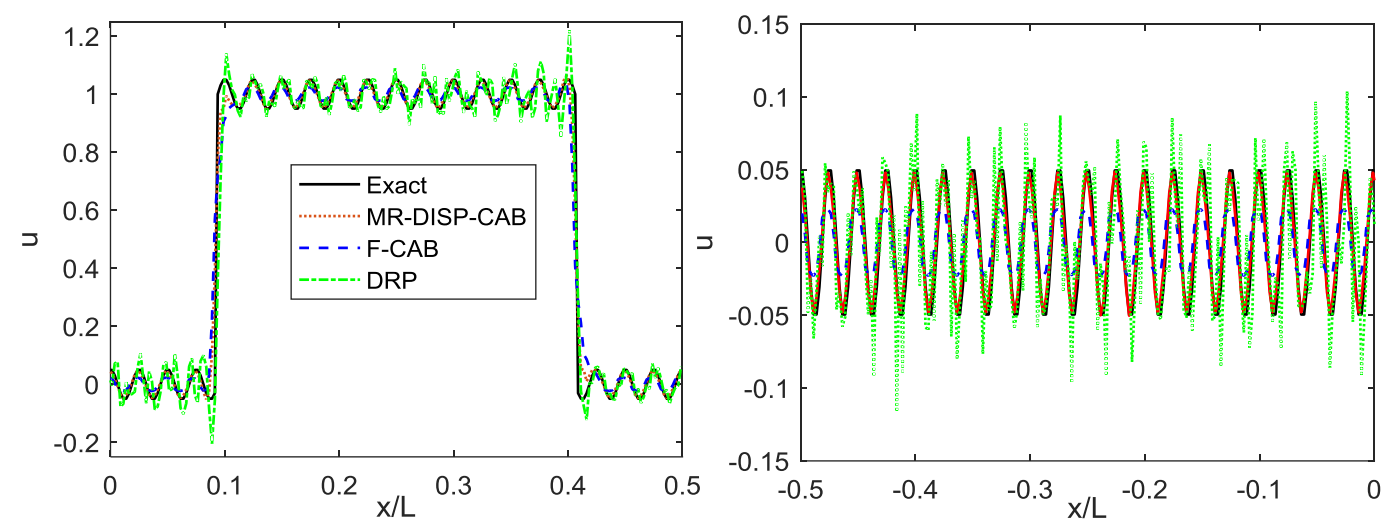

Fig. 12. Linear advection of a rectangular profile appended by a high-frequency wave: the dispersion improved CABARET with the modified relaxed correction (MR-DISP-CAB) against the standard CABARET solution with the full correction (F-CAB) and the DRP scheme. 


\begin{tabular}{|l|l|l|l|l|}
\hline grid size & MR-CAB-DISP & order & DRP & order \\
\hline 6400 & $8.50 \mathrm{E}-04$ & 0.69 & $7.90 \mathrm{E}-03$ & 0.51 \\
\hline 3200 & $1.37 \mathrm{E}-03$ & 0.68 & $1.12 \mathrm{E}-02$ & 0.54 \\
\hline 1600 & $2.20 \mathrm{E}-03$ & 0.66 & $1.63 \mathrm{E}-02$ & 0.53 \\
\hline 800 & $3.47 \mathrm{E}-03$ & 0.65 & $2.35 \mathrm{E}-02$ & 0.51 \\
\hline 400 & $5.46 \mathrm{E}-03$ & 0.64 & $3.36 \mathrm{E}-02$ & 0.4 \\
\hline 200 & $8.51 \mathrm{E}-03$ & & $4.44 \mathrm{E}-02$ & \\
\hline
\end{tabular}

Table 2. Grid convergence for the dispersion- improved CABARET scheme with the modified relaxed correction (MR-DISP-CAB) and the DRP scheme in the linear advection test of a rectangular profile appended by a high-frequency wave.

Next, the linear advection test proposed by Jiang and Shu [29] is considered. In the latter publication, the initial profile that is composed of a Gaussian, a square wave, a triangle wave, and a half ellipse shape according to

$$
u(x, 0)=\left\{\begin{array}{lr}
\frac{1}{6}\left(e^{-\beta(x / L+0.695)}+e^{-\beta(x / L+0.705)}+4 e^{-\beta(x / L+0.7)}\right), & -0.8 \leq x / L \leq-0.6, \\
1, & -0.4 \leq x / L \leq-0.2, \\
1-|10(x / L-0.1)|, & 0 \leq x / L \leq 0.2, \\
\frac{1}{6}(\psi(x / L, 0.495)+\psi(x / L, 0.505)+4 \psi(x / L, 0.5)), & 0.4 \leq x / L \leq 0.6, \\
0, & \text { otherwise },
\end{array}\right.
$$

where $\beta=\log 2 /\left(9 \times 10^{-4}\right), \psi\left(x / L, x_{0} / L\right)=\sqrt{\max \left(1-100\left(x / L-x_{0} / L\right)^{2}, 0\right)}$, is considered in a periodic solution domain at control time $t=8$ and uniform grid consisting of 200 cells to test the performance of several fourth- and fifth-order ENO and WENO Roe schemes.

Here we compare performance of the dispersion-improved CABARET scheme with the relaxed modified flux correction at $\varepsilon=0.2$ and the standard CABARET with the full flux correction on the same test.

Fig.13 shows the corresponding CABARET solutions at $\mathrm{CFL}=0.2$ and the analytical one for reference. Compared to the standard CABARET with the full flux correction, the dispersionimproved CABARET scheme performs much better in less smearing the discontinuities and sharp peaks as well as in better capturing the solution phase which effect is particularly noticeable for the first narrow Gaussian part of the distribution. It can also be noted that the 
overall accuracy of the dispersion-improved CABARET scheme for this problem is comparable to that of the solution corresponding to the $4^{\text {th }}$ ENO- and the $4^{\text {th }}$ and the $5^{\text {th }}$ order WENO-Roe schemes at CFL=0.4 (see figs.2a,b,c from [29]).

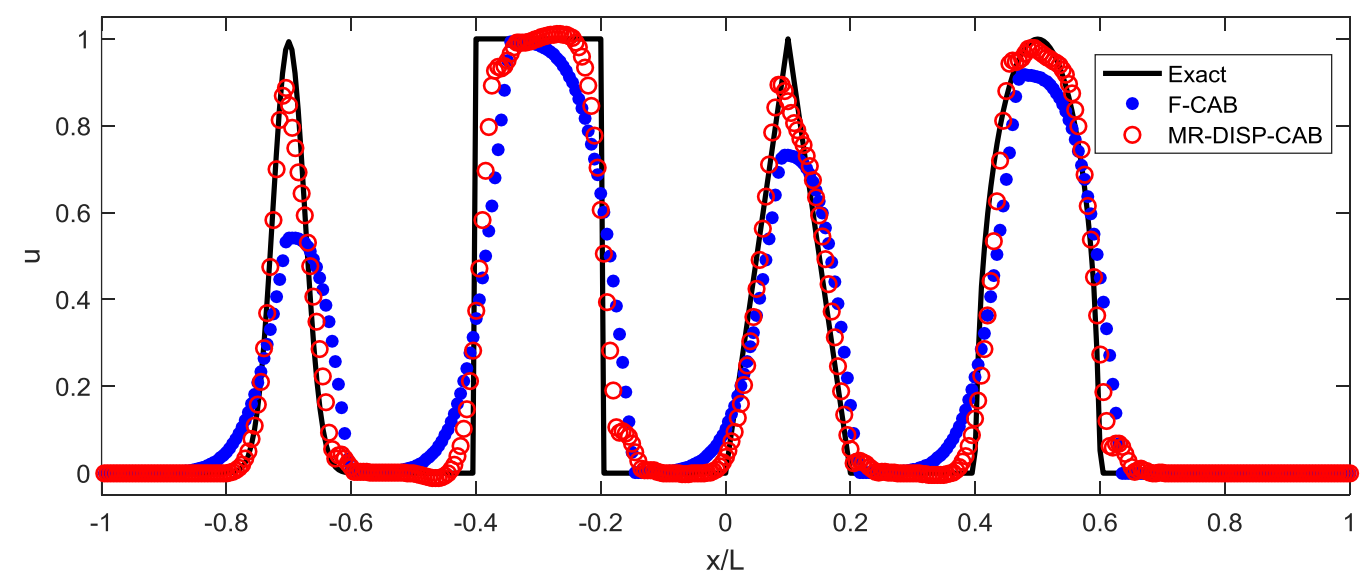

Fig. 13. Comparison of the dispersion-improved CABARET scheme with the modified relaxed flux correction and the standard CABARET scheme with the full flux correction in the linear advection test case from Jiang and Shu [29].

\subsection{D linear advection}

The two-dimensional linear advection equation is considered next which is a limiting case of (18) in case of $2 \mathrm{D}$ scalar equation when $\mathbf{U} \rightarrow u, \mathbf{F} \rightarrow c_{x} u, \mathbf{G} \rightarrow c_{y} u, \mathbf{H}=0$. The problem is solved in a square Cartesian domain with periodic boundary conditions in the $\mathrm{x}$ and $\mathrm{y}$ directions. For numerical solution with the CABARET methods outlined in section 4, the domain is covered by a uniform rectangular grid with equal spacings in the $\mathrm{x}$ and $\mathrm{y}$ directions. The solution is obtained for the velocity field $\left(c_{x}, c_{y}\right)=(1,1)$ corresponding to the translation of the initial profile at $45^{\circ}$ angle to the grid lines.

The initial condition corresponds to

$u(x, 0)=\exp \left(-\frac{x^{2}+y^{2}}{\sigma^{2}}\right), \sigma=0.2 L,-1 \leq x / L \leq 1,-1 \leq y / L \leq 1$.

For this test, computational grid $(50 \times 50)$ is considered which corresponds to resolving the half-width of the Gaussian on 5 grid cells. Two computational schemes are considered: the standard CABARET method with the full flux correction and the dispersion-improved CABARET method with the modified relaxed correction at $\varepsilon=0.2$. For both methods, CFL numbers in the $\mathrm{x}$ - and y-direction are fixed so that $C F L_{x}=C F L_{y}=0.1$.

Figure 14 compares the two solutions obtained with at the control time corresponding to 5 full cycles of the profile crossing the solution domain, $t=5 T$, where $T=2 L$. The dispersionimproved CABARET solution remains in a very good agreement with the analytical solution 
compared to the standard CABARET which shows a phase offset as well as some $30 \%$ loss of the peak amplitude.

Figure 15 presents a $3 \mathrm{D}$ view of the time evolution of the initial profile with the two CABARET schemes: the initial condition, the solution after 5 full cycles, $t=5 T$, and the solution after 10 full cycles, $t=10 T$. It can be seen that compared to the standard CABARET solution the dispersion improved CABARET preserves the shape of the initial profile very well at all times.

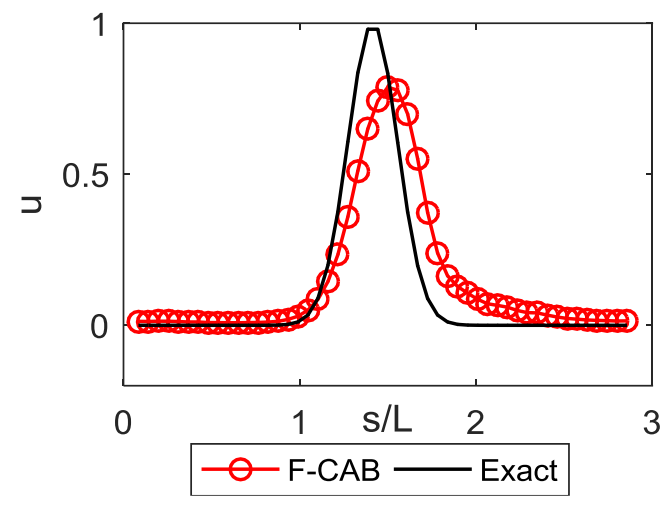

(a)

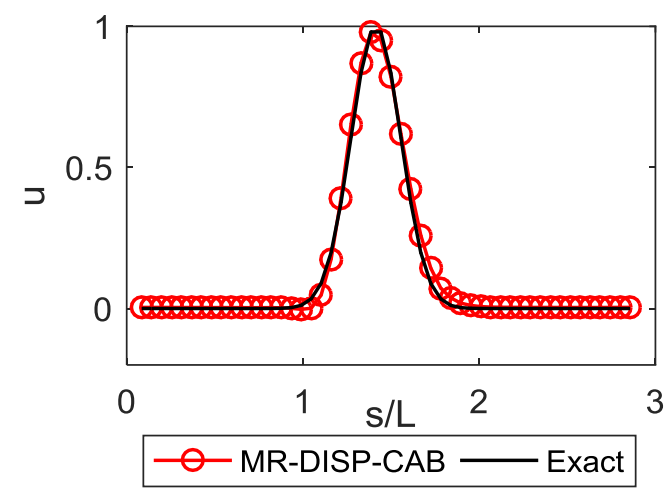

(b)

Fig.14. Linear advection of a 2D Gaussian profile at $45^{\circ}$ angle to the grid axis: (a) 1D slice of the standard CABARET solution with the full correction (F-CAB) along the diagonal direction aligned with the profile propagation and (b) the same for the dispersion-improved CABARET method with the modified relaxed correction at $\varepsilon=0.2$ (MR-DSIP-CAB). 

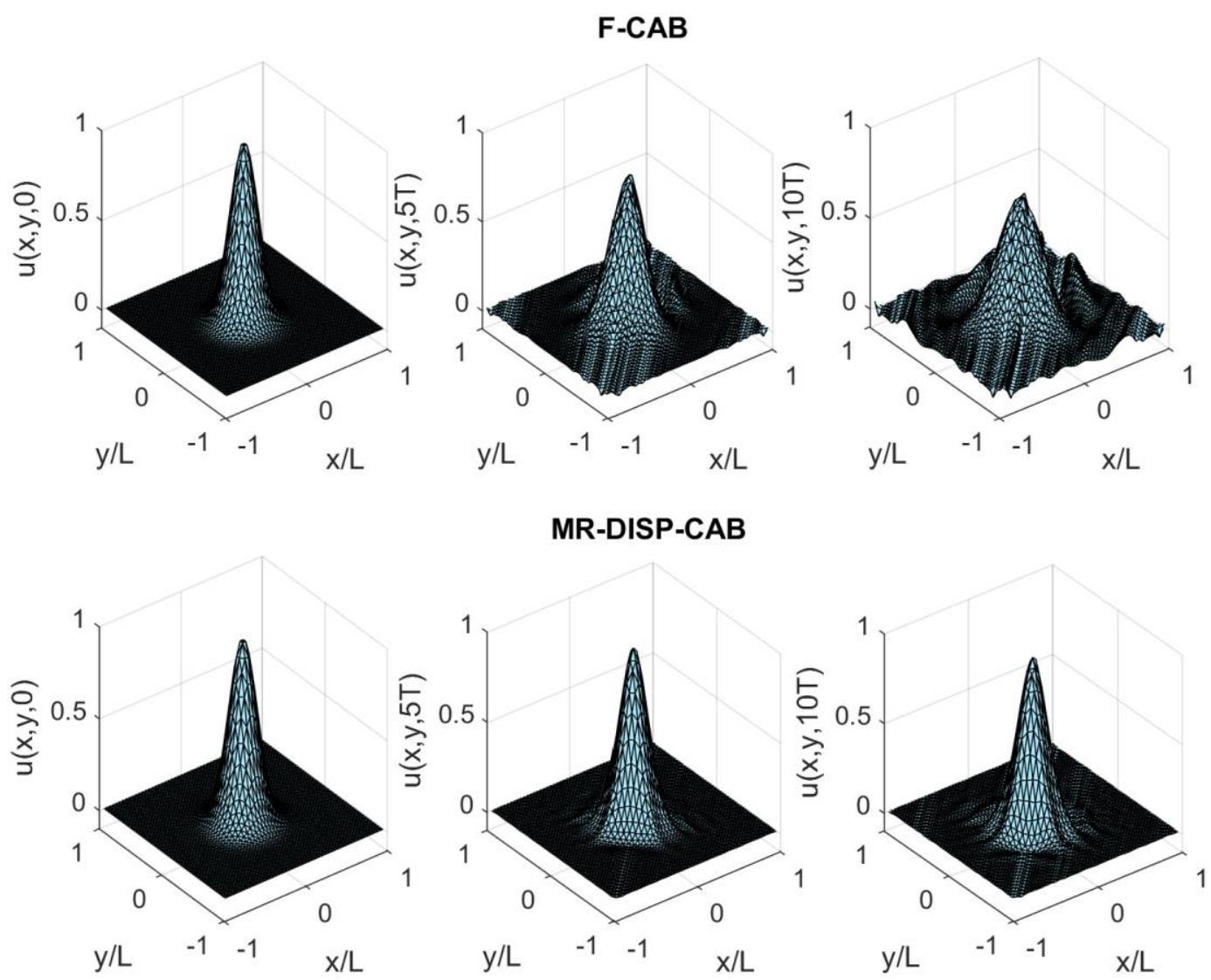

Fig. 15. Time evolution of the 2D Gaussian profile when it is advected at $45^{\circ}$ angle to the grid lines: the standard CABARET solutions with the full correction (F-CAB) against the dispersion-improved CABARET solutions with the modified relaxed correction at $\varepsilon=0.2$ (MR-DSIP-CAB).

\subsection{D Inviscid Burgers' equation}

The numerical solution of the inviscid Burger's equation is considered next, which corresponds to assuming $f=u^{2} / 2$ in the governing scalar conservation law [1]. The following initial condition in a periodic solution domain is used

$$
\begin{aligned}
& u(x, 0)=\sin (2.5 \pi x / L), 0 \leq x \leq 0.4 L, \\
& u(x, 0)=0,0.4 L<x \leq L .
\end{aligned}
$$

The CFL number is assumed to be 0.2 and the adaptive time step is used that depends on the local current velocity so that $\Delta t^{n}=C F L / \max _{1 \leq i \leq N}\left(u_{i+1 / 2}^{n}\right)$, where cell indices 1 and $\mathrm{N}$ correspond to the boundary cells.

The above problem is computed with the standard and the dispersion-improved CABARET schemes with the full baseline flux correction (10) and the modified relaxed correction (12) at $\varepsilon=0.2$ without any anti-relaxation in the shock region (13), (14.1). 
Two other solutions considered correspond to the dispersion improved CABARET scheme with the dispersion term limiter (15)-(17) at $\alpha=1$ and $\alpha=2$ which is always active without using the switch-on condition (15.1).

All calculations were performed with the grid resolution of 80 grid cells and the reference solutions correspond to 800 cells. The control time is $t=4000$. Figures 16 and 17 show comparisons of the different solutions for the case of the full flux correction and the modified relaxed correction, respectively.

First, either with the full flux correction or with the modified relaxed correction, the standard CABARET solution on the fine grid, which is used as the reference, captures the same shock position at the given control time without notable oscillations. The fine grid flux-corrected dispersion improved CABARET solution (not shown) converges to the same result.

The dispersion-improved CABARET solution without limiting the dispersion term show some overshoot of the upstream shock state, which results in an offset shock position compared to the reference solution. The use of the dispersion term limiter reinstates the correct location of the shock in accordance with the reference solution within the half-width of the shock on the coarse grid, where the shock is captured approximately over 3 cells.

For the full flux correction, some very marginal post-shock oscillations appear in the solution with the dispersion term limiter at the maximum truncation threshold value $\alpha=2$, which seem to be completely absent at $\alpha=1$. For the modified relaxed flux correction, the coarse grid dispersion-improved CABARET solutions at $\alpha=1$ show some notable numerical oscillations downstream of the shock, which become small for the limiter threshold at $\alpha=2$.

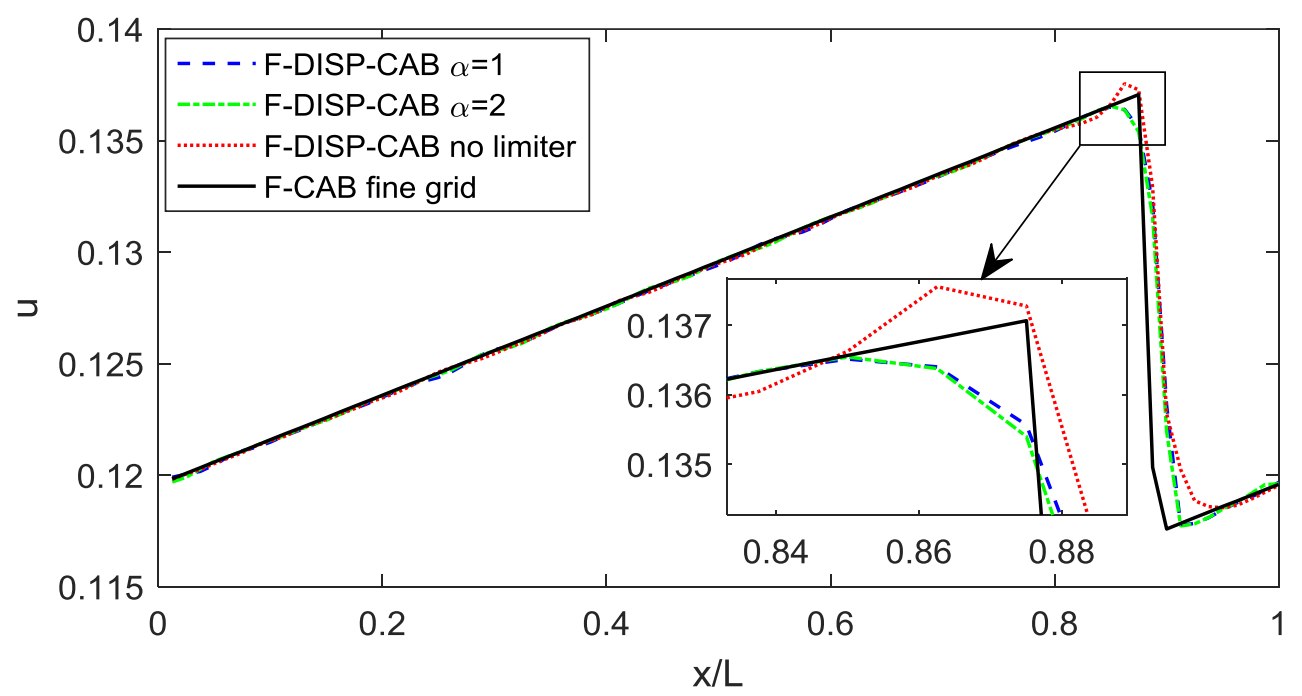

Fig. 16. Solutions to the inviscid Burger's problem of non-linear wave propagation using the dispersion improved CABARET with the full flux correction: no dispersion term limiter (FDISP-CAB no limiter), with the dispersion term limiter at $\alpha=1$ (F-DISP-CAB $\alpha=1$ ), and with the dispersion term limiter at $\alpha=2$ (F-DISP-CAB $\alpha=2$ ) against the reference fine grid solution (F-CAB fine grid). 


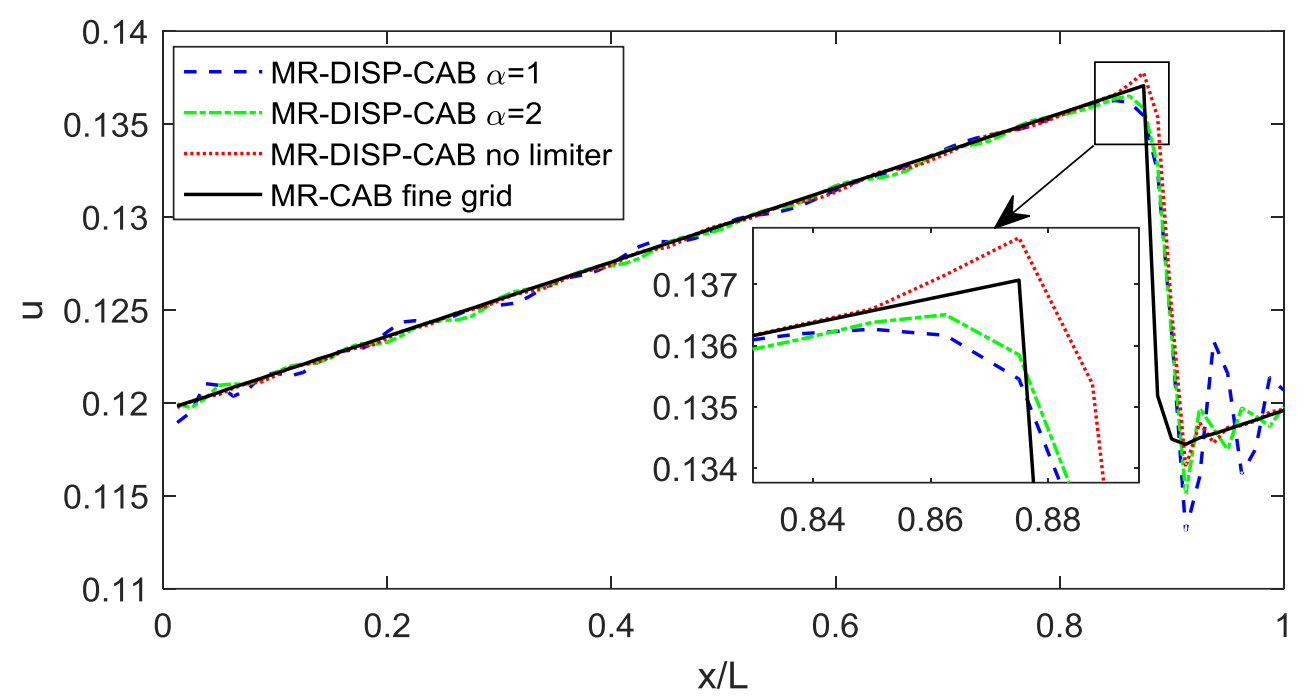

Fig. 17. Solutions to the inviscid Burger's problem of non-linear wave propagation using the dispersion improved CABARET with the modified relaxed flux correction at $\varepsilon=0.2$ : no dispersion term limiter (MR-DISP-CAB no limiter), with the dispersion term limiter at $\alpha=1$ (MR-DISP-CAB $\alpha=1$ ), and with the dispersion term limiter at $\alpha=2$ (MR-DISP-CAB $\alpha=2$ ) against the reference fine grid solution (MR-CAB fine grid).

\subsection{D and 2D isothermal gas dynamics}

Pressure wave propagation in gas dynamics in accordance with the governing equations (26) is considered next in a rectangular solution domain $-1 \leq x / L_{x} \leq 1,-1 \leq y / L_{y} \leq 1$ with the constant background flow $(\rho, u, v)=\left(\rho_{0}, U, 0\right)$, where $M=U / c=0.5$. Periodic boundary conditions are assumed in the $y$-direction, the outflow boundary conditions are specified at the outlet, $x / L_{x}=1$ corresponding to a characteristic non-reflecting boundary condition, and the inflow condition corresponding to the incoming pressure wave is specified at the inlet, $x / L_{x}=-1$.

The incoming wave boundary condition is set up in accordance with the following linear propagation wave solution. Let's consider (26) which are linearised about the background flow in accordance with $(\rho, u, v)=\left(\rho_{0}+\rho^{\prime}, U+u^{\prime}, v^{\prime}\right)$, where the prime indicates fluctuations from the background values. The resulting linear gas dynamics equations can then be re-arranged to the convective wave propagation equation with respect to the density fluctuation:

$$
\left(\frac{\partial}{\partial t}-U \frac{\partial}{\partial x}\right)\left(\frac{\partial}{\partial t}-U \frac{\partial}{\partial x}\right) \rho^{\prime}-c^{2} \frac{\partial^{2}}{\partial x \partial x} \rho^{\prime}-c^{2} \frac{\partial^{2}}{\partial y \partial y} \rho^{\prime}=0
$$


The above equation admits periodic wave solutions in the form of $\rho^{\prime}=\varepsilon_{0} \rho_{0} \cdot e^{i\left(\omega t-k_{x} x-k_{y} y\right)}$ where $0<\varepsilon_{0}<<1$ which satisfy the following dispersion relation

$$
\omega^{2}+2 k_{x} U+k_{x}^{2} U^{2}-c^{2} k_{x}^{2}-c^{2} k_{y}^{2}=0 .
$$

From the above relation, for each specified wave frequency, $\omega$ and the wave angle between the front normal and the flow direction, $\eta=k_{y} / k_{x}=\tan (\theta)$, the following equations for the wavenumbers $k_{x}$ and $k_{y}$ can be obtained:

$$
k_{x}=\frac{\omega}{c} \frac{\sqrt{1-\eta}-M}{1-M^{2}}, \quad k_{y}=\frac{\omega}{c} \frac{\sqrt{\eta}}{\sqrt{1-M^{2}}} .
$$

In particular, (57) shows that the condition of cut-on waves is $0 \leq \eta<1$ where $\eta=0$ corresponds to the one-dimensional (normal) wave propagation.

The corresponding analytical solution for the propagation wave then is

$$
\begin{aligned}
& \rho(x, y, t)=\rho_{0}\left(1+\varepsilon_{0} \cdot e^{i\left(\omega t-k_{x} x-k_{y} y\right)}\right) \\
& u(x, y, t)=U\left(1+\varepsilon_{0} \cdot \frac{c^{2}}{U} k_{x} e^{i\left(\omega t-k_{x} x-k_{y} y\right)}\right) /\left(\omega-k_{x} U\right) \\
& v(x, y, t)=U\left(1+\varepsilon_{0} \cdot \frac{c^{2}}{U} k_{y} e^{i\left(\omega t-k_{x} x-k_{y} y\right)}\right) / \omega
\end{aligned}
$$

which also satisfies the periodic boundary conditions in the $y$-direction if the height of the solution domain is adjusted so that $L_{y}=\pi / k_{y}$.

In the numerical examples which follow first, a small amplitude normal wave $(\theta=0)$ at $\varepsilon_{0}=10^{-5}$ satisfying (59) is imposed at the inlet. Then, the full nonlinear governing equations (26) are solved numerically with the following methods: the original CABARET scheme and the dispersion improved CABARET scheme with either the full baseline (10) or the modified relaxed flux correction (12). For the linear wave propagation, both uniform and non-uniform grids are used with the variable grid resolution, which correspond to 8 points per wavelength $(p p w)$ for the uniform grid and 6-40 ppw for the non-uniform grid test case. The CFL number is 0.1 for all uniform grid tests and is set to 0.8 for the non-uniform grid test. In the latter case, the grid resolution increases from the boundaries to the domain centre so that the CFL number in the coarse part of the grid near the inlet and the outlet boundary is also approximately equal to 0.1 .

The results of the normal wave propagation test case are summarised in Figs. 18-22. It can be seen that the standard CABARET scheme with the full baseline flux correction (10) quickly dissipates the high frequency wave (Fig.18). Note that the analytical solution for this test and several other linear wave tests in this section appears choppy because of missing the crests and the troughs of the wave on the coarsest grids used. The results of the dispersion-improved CABARET scheme used with full flux correction (not shown) are very similar to these. The modified relaxed-flux correction at the relaxation parameter $\varepsilon=0.2$ (12) works very well for preserving the wave amplitude but the phase error of the standard CABARET solution remains very pronounced (Figs.19, 21). The application of the dispersion improved CABARET results 
with modified relaxed-flux correction at the relaxation parameter $\varepsilon=0.2$ leads to a good preservation of both the solution amplitude and the phase (Figs. 20, 22).

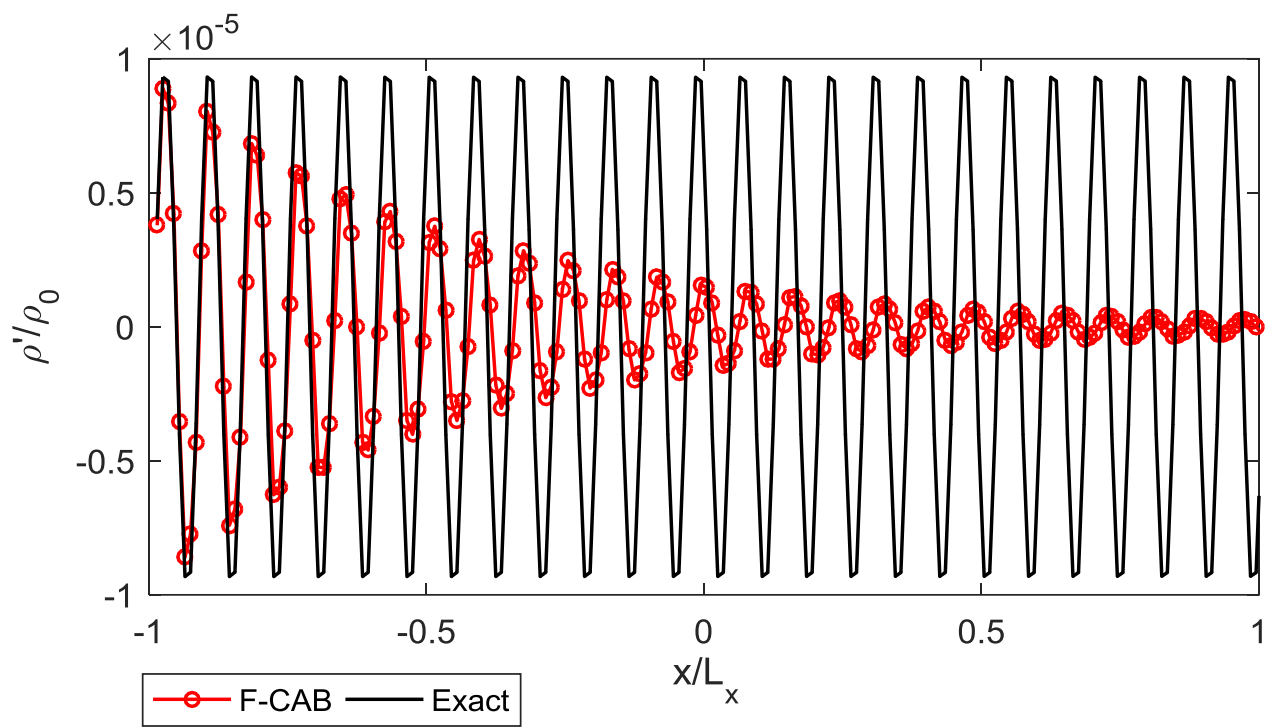

Fig. 18. The dissipative effect of the standard CABARET correction with the full correction (F-CAB) for a small amplitude normal wave propagation in uniform flow for a uniform numerical wave resolution at 8 points per wavelength.

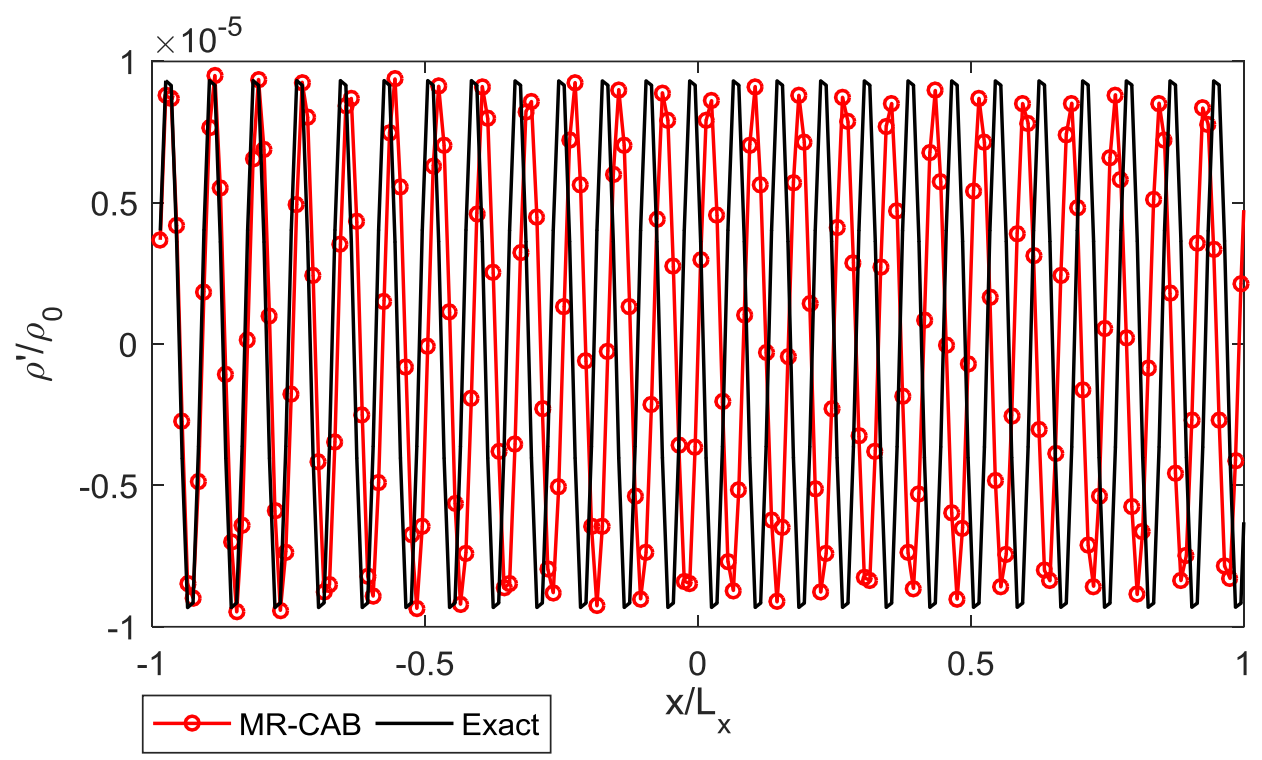

Fig. 19. The dispersive effect of the standard CABARET with the modified-relaxed correction algorithm (MR-CAB) at $\varepsilon=0.2$ for a small amplitude normal wave propagation in uniform flow for a uniform numerical wave resolution at 8 points per wavelength. 


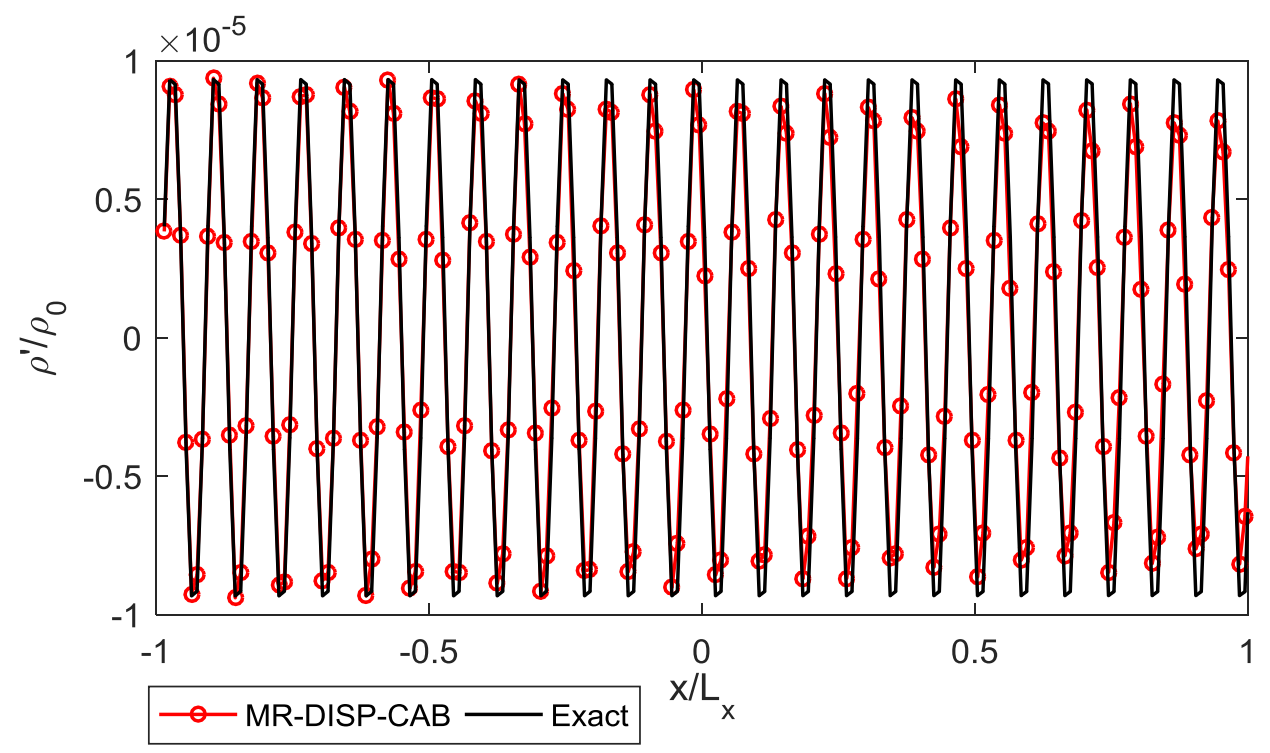

Fig. 20. Dispersion improved CABARET solution with the modified-relaxed correction algorithm (MR-DISP-CAB) at $\varepsilon=0.2$ for a small amplitude normal wave propagation in uniform flow for a uniform numerical wave resolution at 8 points per wavelength.

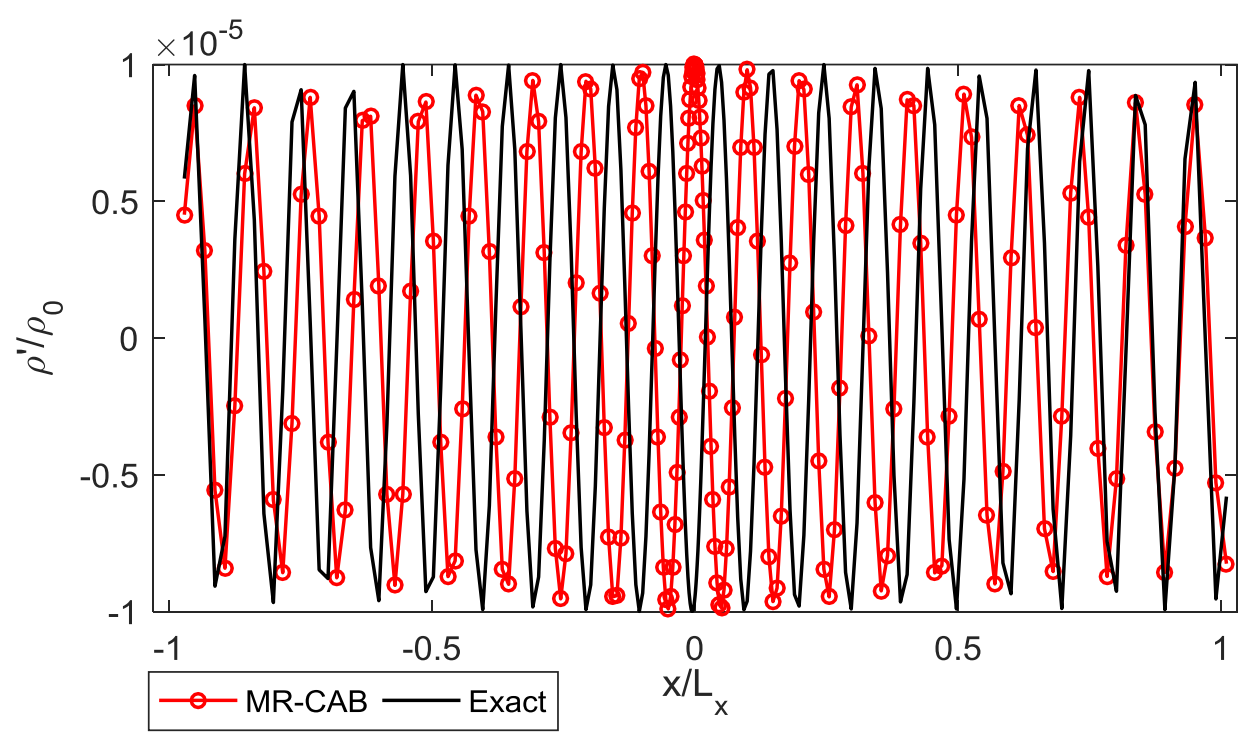

Fig. 21. The dispersive effect of the standard CABARET with the modified-relaxed correction algorithm (MR-CAB) at $\varepsilon=0.2$ for a small amplitude normal wave propagation in uniform flow for a non-uniform numerical wave resolution that grows from 6 points per wavelength near the inlet/outlet boundaries to 40 points per wavelength in the centre of the computational domain. 


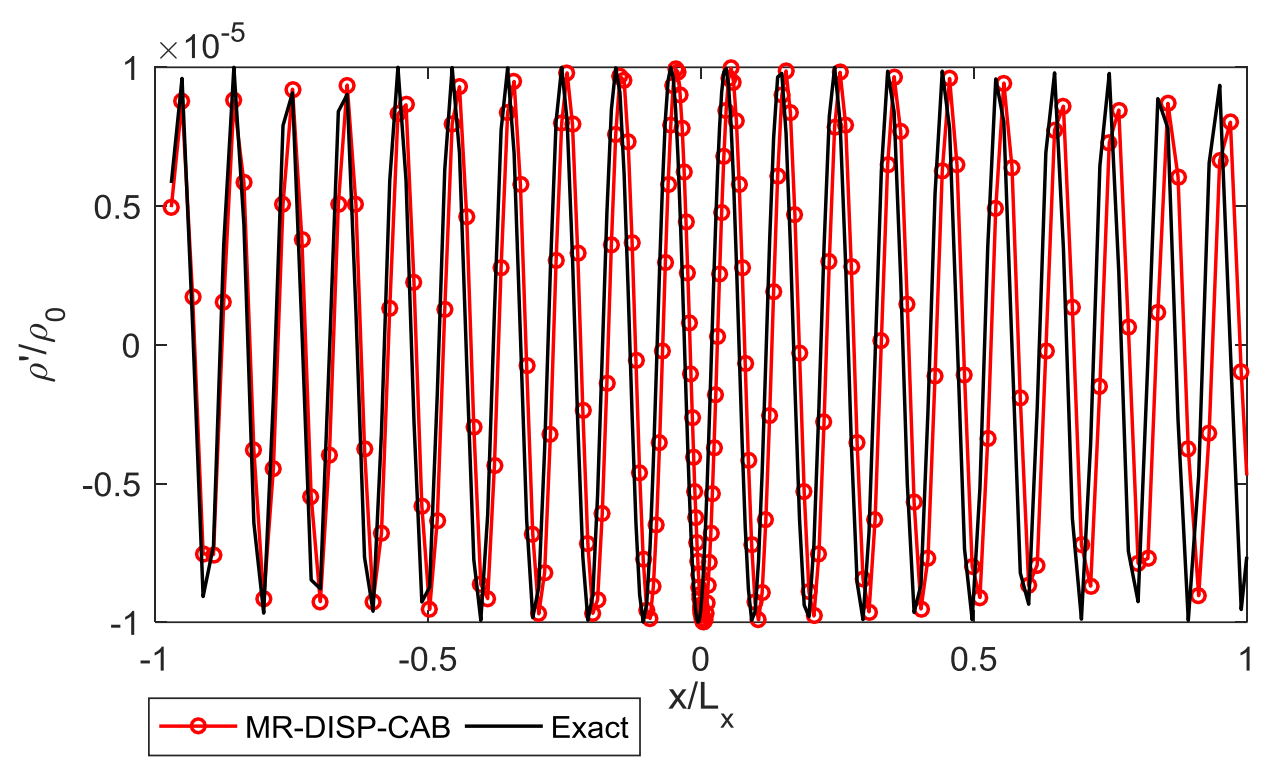

Fig. 22. Dispersion improved CABARET solution with the modified-relaxed correction algorithm (MR-DISP-CAB) at $\varepsilon=0.2$ for a small amplitude normal wave propagation in uniform flow for a non-uniform numerical wave resolution that grows from 6 points per wavelength near the inlet/outlet boundaries to 40 points per wavelength in the centre of the computational domain.

Next, a further challenging problem is considered: a small amplitude oblique wave propagating at angle $\theta=58^{\circ}$ to the flow, which approaches the wave cut-off range $\left(\theta=90^{\circ}\right)$. The amplitude of the wave is the same as considered in the previous tests. A rectangular domain is considered that is covered by a uniform grid at the resolution corresponding to $15 p p w$ in the $x$-direction and $9 p p w$ in the y-direction is used. The CFL number in the $x$-direction is 0.1 .

Likewise for the normal wave propagation problem, the full non-linear governing equations (26) are solved with the same CABARET schemes. The results of the oblique wave propagation test are summarised in Figs. 23-25. First, in case of the CABARET with the full baseline correction (10), even a twice higher grid resolution in the $\mathrm{x}$-direction compared to the normal wave propagation leads to a similar strong dissipation of the wave amplitude (compare Fig. 23 with Fig. 18). For the oblique wave propagation test, best results in terms of preserving the high-frequency solution amplitude are obtained with the relaxed flux correction method (12) where the relaxation parameter is set to the maximum range, $\varepsilon=0.4$. Again, even with the amplitude preserved, the standard CABARET methods still leads to a prominent phase error (Fig. 24) while the dispersion improved CABARET leads to very acceptable results compared to the analytical solution (Fig. 25). 


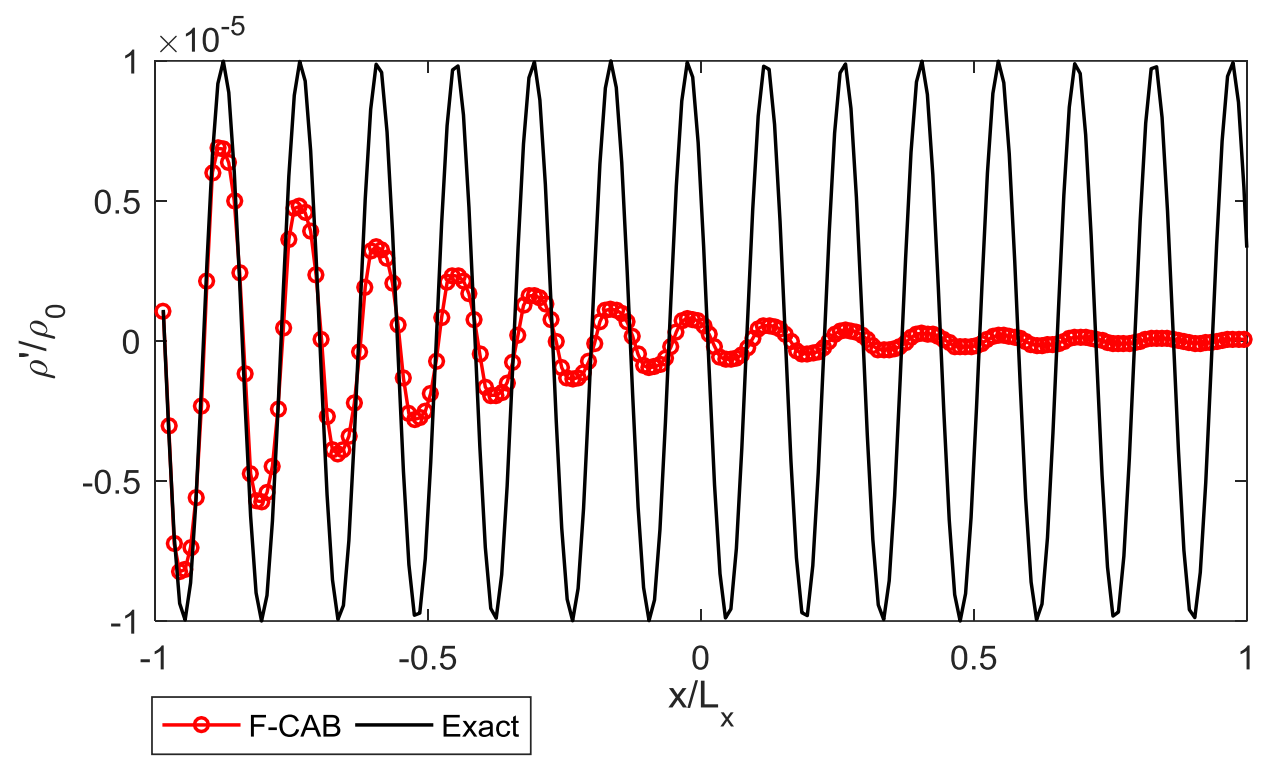

Fig. 23. The dissipative effect of the standard CABARET correction with the full correction (F-CAB) for a small amplitude 2D oblique wave propagation at $58^{0}$ angle to the uniform flow for a uniform numerical wave resolution at 15 and 9 points per wavelength in the $x$ - and $y$ direction, respectively.

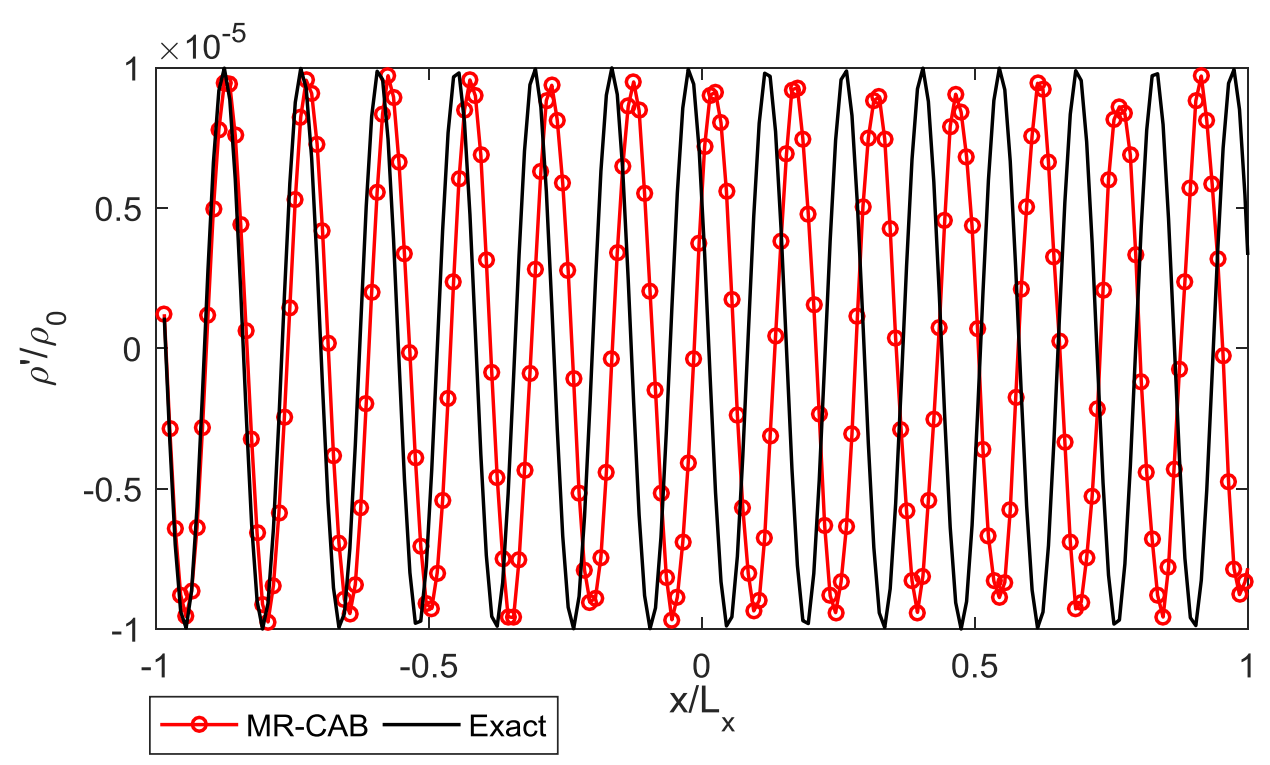

Fig. 24. The dispersive effect of the standard CABARET with the modified-relaxed correction algorithm (MR-CAB) at $\varepsilon=0.4$ for a small amplitude 2D oblique wave propagation at $58^{0}$ angle to the uniform flow for a uniform numerical wave resolution at 15 and 9 points per wavelength in the $x$ - and $y$-direction, respectively. 


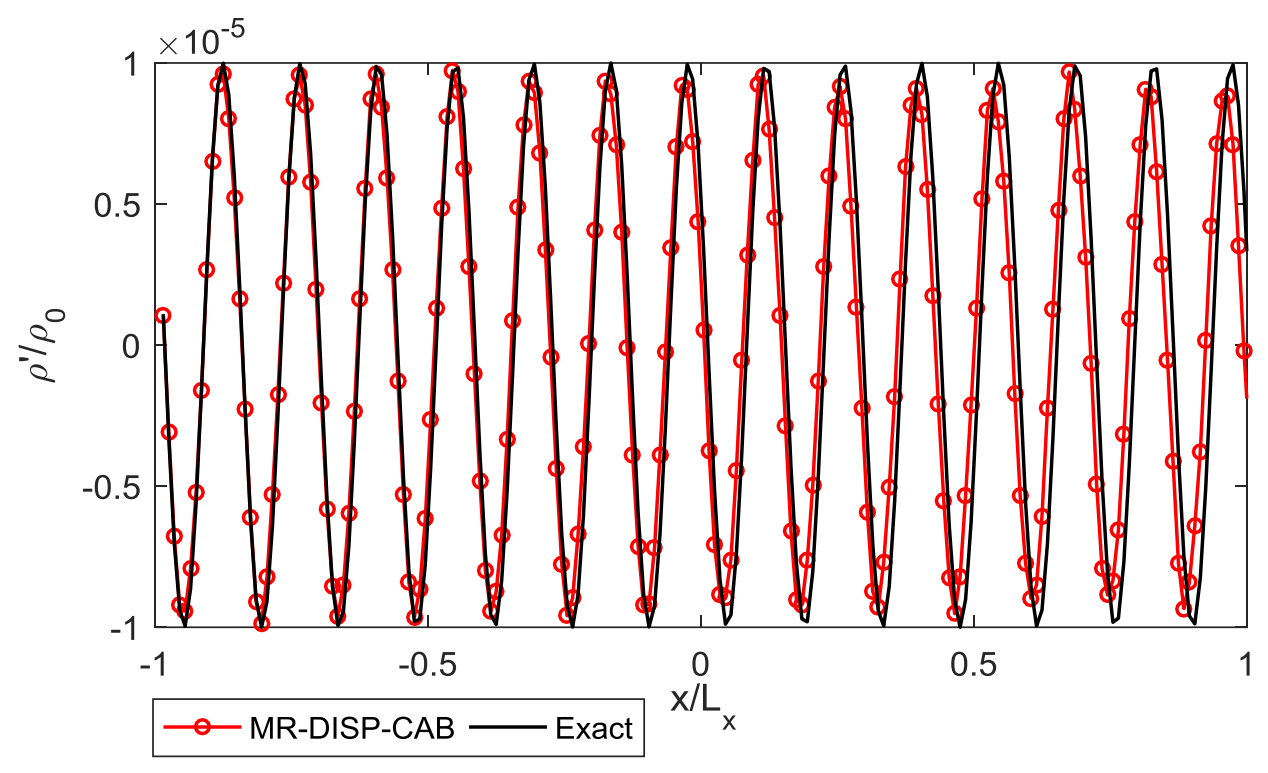

Fig. 25. Dispersion improved CABARET solution with the modified-relaxed correction algorithm (MR-DISP-CAB) at $\varepsilon=0.4$ for a small amplitude $2 \mathrm{D}$ oblique wave propagation at $58^{0}$ angle to the uniform flow for a uniform numerical wave resolution at 15 and 9 points per wavelength in the $x$ - and $y$-direction, respectively.

Finally, a nonlinear wave propagation is considered by specifying a large amplitude normal wave corresponding to $\varepsilon_{0}=0.5$ in (59) as the inlet condition for the same nonlinear governing equations (26). In this case, the analytical solution based on linear wave propagation equation (56) is no longer valid and the reference numerical solution is obtained using the standard CABARET method with the full flux correction at the fine grid resolution. The reference solution corresponds to the control time moment $t=15(\mathrm{~L} / \mathrm{c})$ when the initial periodic wave imposed at the inlet boundary develops a $z$-wave profile due to the non-linear steepening process since the wave crest travels faster than the trough. For the fine grid resolution considered, which corresponds to $2500 \mathrm{ppw}$ of the incoming wave solution, the fine-grid solution can be regarded as virtually converged compared to the coarse grid CABARET solutions at $100 \mathrm{ppw}$ which will be compared and contrasted with the reference solution.

The following schemes are compared: (i) the standard CABARET scheme with the full baseline correction method (10) and (ii) the dispersion improved CABARET scheme with the non-linear limiter function (13), (14.1) at $\varepsilon=0.2$. The latter flux limiter leads to the full baseline flux correction (10) activated in the shock region while preserving the modified relaxed flux correction (12) in the small amplitude wave region (away from the shock). In addition to the above, the third solution considered corresponds to the scheme from (ii) but where the non-linear limiter algorithm for the dispersion term (15)-(17) is also active. The latter condition allows truncating the dispersion term if the non-linear wave condition (15.1) is satisfied, e.g. near the shock.

Numerical results for the coarse grid solutions of the standard CABARET method based on the full flux correction and the dispersion improved CABARET with and without implementing the limiter function for the dispersion term are shown in Fig. 26. It can be seen that the application of the non-linear limiter function for flux correction relaxation with the 
modification for non-linear waves (13), (14.1) leads to clean, numerical oscillation-free solutions in all cases. However, it is only when the dispersion term limiter is also activated the upstream and downstream shock states can be correctly predicted and the position of the shock front is captured by the dispersion improved CABARET solution in excellent agreement with the reference solution (Fig. 26b).

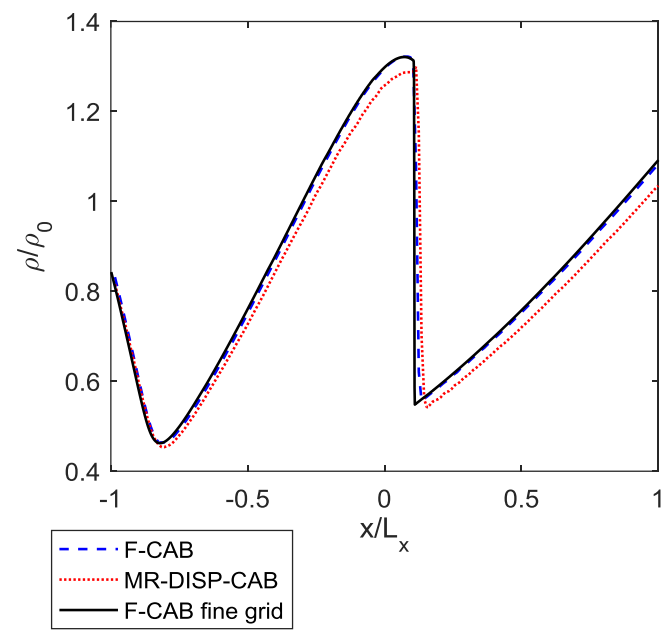

(a)

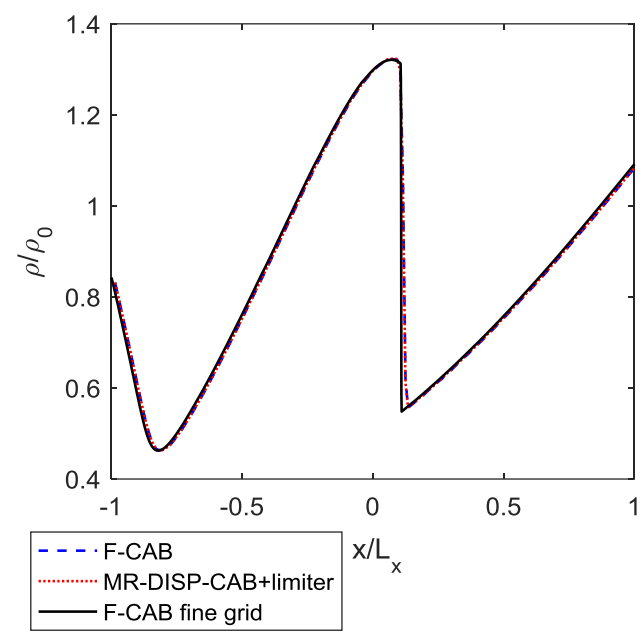

(b)

Fig. 26. Effect of the dispersion term limiter for correctly preserving the shock speed in the high-amplitude non-linear wave propagation problem: F-CAB stands for the standard CABARET with the full flux correction on the coarse or the fine grid, MR-DISP-CAB stands for the dispersion improved CABARET with the modified relaxed flux correction on the coarse grid, and MR-DISP-CAB+limiter stands for the dispersion improved CABARET with the modified relaxed flux correction on the coarse grid with the dispersion term limiter activated in the non-linear flow region.

\section{Conclusions}

A new class of the dispersion-improved CABARET schemes is developed for linear and nonlinear wave propagation problems. The schemes are based on optimising the dispersion properties of the original CABARET method by adding the artificial (anti) dispersion term using cell-face values from the adjacent computational cells, which for gas dynamics problems results in a marginally more extended computational stencil compared to the original CABARET. It is shown how the optimal parameter of the artificial dispersion term can be evaluated analytically. For dealing with discontinuities and non-linear wave problems, several non-linear versions of the dispersion-improved CABARET are introduced based on the flux correction and the limiter for the artificial dispersion term, which can be automatically relaxed or completely deactivated away from the shocks. All these schemes can be viewed as a generalisation of the original three-time-level advection scheme proposed by Goloviznin and Samarskii [6] to the class of two-time-level nonlinear predictor-corrector schemes for solving hyperbolic problems in multiple dimensions.

Dissipation and dispersion properties of the linear and the non-linear dispersion improved CABARET schemes are analysed analytically and numerically, which show superior 
properties compared to the standard CABARET version. Numerical results of the linear advection tests including the $2 \mathrm{D}$ test for linear advection at $45^{\circ}$ angle to the grid lines confirm the same conclusion. For linear advection of a smooth initial profile, the dispersion-improved CABARET schemes with and without the relaxed flux correction show the second-order rate of numerical grid convergence. For a discontinuous profile with a high-frequency wave appended, the dispersion-improved CABARET scheme with the modified relaxed correction captures both the discontinuity and the high-frequency wave compared to the standard CABARET method and the popular fourth-order Dispersion Relation Preserving scheme by Tam and Web [28]. Performance of the dispersion-improved CABARET scheme is also compared with the standard CABARET scheme in the linear advection test from Jiang and Shu [29]. Compared to the standard CABARET, the dispersion-improved CABARET is able to capture sharp discontinuities and peaks rather well - on par with some of the 4th -5th-order ENO and WENO Roe schemes. Linear wave propagations tests in gas dynamics also demonstrate that the dispersion improved CABARET scheme can propagate waves at numerical resolution of 6-8 points per wavelength $(p p w)$ for normal waves and 9-15 $p p w$ for oblique waves at $58^{0}$ angle to the flow direction with a good preservation of the amplitude and the phase of the solution. Finally, for non-linear wave propagation problems, such as the ones which occur when solving the inviscid Burger's equation or the development and the propagation of z-waves in the isothermal gas dynamics, the non-linear dispersion improved CABARET schemes correctly capture the shock location within the half-width of the shock on the coarse grid, where the shock is spread approximately over 3 cells, and without any significant oscillations.

\section{Acknowledgements}

SEN acknowledges the support from Partnership for Observation of Global Oceans and Scientific Committee for Oceanic Research (POGO-SCOR). The authors are also grateful to Prof. Vasily Mihailovich Goloviznin and Dr. Anton Markesteijn for fruitful discussions of the modified flux correction algorithms for the CABARET method implemented in this publication.

\section{References}

[1] Liu, X.D., Osher, S., Chan. T., Weighted essentially non-oscillatory schemes. J. Comput. Phys. 1994; 115.1: 200-212.

[2] Shu, C.W., Osher, S., Efficient implementation of essentially non-oscillatory shockcapturing schemes. J. Comput. Phys. 1988; 77.2: 439-471.

[3] Cockburn, B., Shu, C.W., Runge-Kutta discontinuous Galerkin methods for convectiondominated problems. J. Sci. Comput. 2001; 16.3: 173-261.

[4] Qiu, J., Shu C.W., Runge-Kutta Discontinuous Galerkin Method Using WENO Limiters. SIAM J. Sci. Comput. 2005; 26.3: 907-929.

[5] Bogey, C, de Cacqueray, N., Bailly, C., A shock-capturing methodology based on adaptative spatial filtering for high-order non-linear computations. J. Comput. Phys. 2009; 228.5: 1447-1465. 
[6] Goloviznin, V.M., Samarskii, A.A., Difference approximation of convective transport with spatial splitting of time derivative. Math. Model. 1998; 10:86-100.

[7] Karabasov, S.A., and Goloviznin, V. M., Compact accurately boundary-adjusting highresolution technique for fluid dynamics. J. Comput. Phys. 2009; 228.19: 7426-7451.

[8] Iserles, A., Generalized Leapfrog methods. IMA J. Numer. Anal. 1986; 6. 381-392.

[9] Roe, P.L., Linear bicharacteristic schemes without dissipation. SIAM J. Sci. Comput. 1998; 19: $1405-1427$.

[10] Lax, P., Wendroff, B., Systems of conservation laws. Communications on Pure and Applied mathematics 1960; 13.2: 217-237.

[11] Karabasov, S.A., and Goloviznin, V.M., New efficient high-resolution method for nonlinear problems in aeroacoustics. AIAA journal 2007; 45.12: 2861-2871.

[12] Faranosov, G.A., Goloviznin, V.M., Karabasov, S.A., Kondakov, V.G., Kopiev, V.F., Zaitsev, M.A., CABARET method on unstructured hexahedral grids for jet noise computation. Comput. Fluids 2013; 88: 165-179.

[13] Semiletov, V.A., Yakovlev P.G., Karabasov S.A., Faranosov, G.A., Kopiev V.F., Jet and jet-wing noise modelling based on the CABARET MILES flow solver and the Ffowcs Williams-Hawkings method. Int. J. Aeroacoust. 2016; 15.(6-7): 631-645.

[14] Semiletov, V.A., and Karabasov, S.A., CABARET scheme for computational aero acoustics: extension to asynchronous time stepping and 3D flow modelling. Int. J. Aeroacoust 2014; 13.(3-4): 321-336.

[15] Semiletov, V.A., and Karabasov, S.A., CABARET scheme with conservation-flux asynchronous time-stepping for nonlinear aeroacoustics problems. J. Comput. Phys. 2013; 253: $157-165$.

[16] Markesteijn, A.P., Semiletov, V.A. and Karabasov, S.A., GPU CABARET Solutions for the SILOET Jet Noise Experiment: Flow and Noise Modelling. AIAA 2016-2967, 22nd AIAA/CEAS Aeroacoustics Conference 2016.

[17] Karabasov, S.A., Berloff, P.S., and Goloviznin, V.M., CABARET in the ocean gyres. Ocean Model. 2009; 30.2: 155-168.

[18] Naghibi, S.E., Jalali M.A., Karabasov S., Alam M.-R., Excitation of the Earth's Chandler wobble by a turbulent oceanic double-gyre, Geophys. J. Int. 2017; 209.1: 509-516.

[19] Obabko, A.V., Fischer, P.F., Tautges, T.J., Karabasov, S.A., Goloviznin, V.M., Zaytsev, M.A., Chudanov, V.V., Pervichko, V.A., Aksenova, A.E., CFD validation in OECD/NEA tjunction benchmark, Argonne National Laboratory Report, ANL/NE-11/25.

[20] Kulikov, Y.M., Son, E.E., The CABARET method for a weakly compressible fluid flows in one- and two-dimensional implementations, Journal of Physics: Conference Series 2016; 774.1

[21] Zaitsev, M.A., Karabasov, S.A., CABARET scheme for computational modelling of linear elastic deformation problems, Math. Modelling, in print. 
[22] Hirsch, C., Numerical computation of internal and external flows: The fundamentals of computational fluid dynamics. Butterworth-Heinemann, 2007.

[23] Pirozzoli, S., On the spectral properties of shock-capturing schemes. J. Comput. Phys. 2006; 219.2: 489-497.

[24] Zyuzina, N. A., Ostapenko, V. V. , Monotone Approximation of a Scalar Conservation Law Based on the CABARET Scheme in the Case of a Sign-Changing Characteristic Field. Doklady Mathematics. 2016; 94. 2: 538-542.

[25] Titarev, V. A., Toro, E. F., Analysis of ADER and ADER-WAF schemes. IMA J Numer Anal. 2007; 27.3: 616-630.

[26] Glotov, V.Y., Mathematical modelling of free turbulence based on the maximum principle, 2014, PhD Thesis, Moscow Institute of Nuclear Safety.

[27] Bogey, C., Bailly, C., A family of low dispersive and low dissipative explicit schemes for flow and noise computations, J. Comput. Phys. 2004; 194.1: 194-214.

[28] Tam, C.K.W., Webb, J.C., Dispersion-Relation-Preserving Finite Difference Schemes for Computational Acoustics, J. Comput. Phys. 1993; 107.2:262-281.

[29] Jiang, G. S., Shu, C. W., Efficient implementation of weighted ENO schemes. J. Comput. Phys. 1996; 126.1: 202-228. 